\title{
Determining $c_{0}$ in $C(\mathcal{K})$ spaces
}

\author{
by
}

\section{S. A. Argyros and V. Kanellopoulos (Athens)}

\begin{abstract}
For a countable compact metric space $\mathcal{K}$ and a seminormalized weakly null sequence $\left(f_{n}\right)_{n}$ in $C(\mathcal{K})$ we provide some upper bounds for the norm of the vectors in the linear span of a subsequence of $\left(f_{n}\right)_{n}$. These bounds depend on the complexity of $\mathcal{K}$ and also on the sequence $\left(f_{n}\right)_{n}$ itself. Moreover, we introduce the class of $c_{0}$-hierarchies. We prove that for every $\alpha<\omega_{1}$, every normalized weakly null sequence $\left(f_{n}\right)_{n}$ in $C\left(\omega^{\omega^{\alpha}}\right)$ and every $c_{0}$-hierarchy $\mathcal{H}$ generated by $\left(f_{n}\right)_{n}$, there exists $\beta \leq \alpha$ such that a sequence of $\beta$-blocks of $\left(f_{n}\right)_{n}$ is equivalent to the usual basis of $c_{0}$.
\end{abstract}

1. Introduction. The present paper is closely related to a recent paper by I. Gasparis, E. Odell and B. Wahl [GOW] and belongs to the area which could be named "effective" infinite-dimensional Banach space theory. Many results in Banach space theory happen to be of a very existential form. An effective approach is to find recursively defined hierarchies which determine the desired property to a certain extent. To make this more transparent let us recall a result from $[\mathrm{AMT}]$ (see also $[\mathrm{AG}],[\mathrm{AGR}]$ ).

Let $\left(x_{n}\right)_{n}$ be a seminormalized weakly null sequence. Then Mazur's theorem shows that there exists a convex block subsequence norm converging to zero. The effective approach to this result is as follows:

TheOREM I. For each countable ordinal $\zeta$ and $L \in[\mathbb{N}]$ there exists a regular summability method denoted by $\zeta^{L}$ such that given a weakly null sequence $\left(x_{n}\right)_{n}$ the following hold:

(a) For each $\zeta<\omega_{1}$ there exists $N \in[\mathbb{N}]$ such that one of the following two alternatives holds:

(i) For all $L \in[N]$, the subsequence $\left(x_{n}\right)_{n \in L}$ is an $l_{1}^{\zeta}$-spreading model.

(ii) For all $L \in[N]$, the sequence $\left(x_{n}\right)_{n}$ is $\zeta^{L}$-(norm) summable.

2000 Mathematics Subject Classification: 46B03, 06A07.

Key words and phrases: $c_{0}$-sequences, Schreier families, $C(\mathcal{K})$ spaces.

Research supported by a grant of EPEAEK program "Pythagoras". 
(b) There exists $\zeta_{0}<\omega_{1}$ such that for all $\zeta<\zeta_{0}$ (resp. $\left.\zeta \geq \zeta_{0}\right)$ the first (resp. the second) alternative of the above occurs.

It is obvious that effective results provide a further understanding of the relevant properties and frequently are key ingredients in applications. Passing to Banach spaces containing $c_{0}$ one could ask the following.

Problem. Let $X$ be a $c_{0}$ saturated Banach space. Does there exist an effective process which for every seminormalized weakly null sequence $\left(x_{n}\right)_{n}$ of $X$ determines a block sequence of $\left(x_{n}\right)_{n}$ equivalent to the usual basis of $c_{0}$ ?

A consequence of the results of [GOW] is a positive answer to the above problem in some special cases. The most important is the case of $C(\mathcal{K})$ with $\mathcal{K}$ a countable compact set. As is well known these spaces are $c_{0}$-saturated $[\mathrm{BP}],[\mathrm{PS}]$. The approach of Gasparis-Odell-Wahl to the solution of the problem goes as follows. First to a given Schauder basic sequence $\left(x_{n}\right)_{n}$ using iteration they assign certain normalized block sequences $\left(\zeta_{n}^{L}\right)_{n}$ with $\zeta<\omega_{1}$ and $L \in[\mathbb{N}]$. They call these the normalized $\zeta$-averages of $\left(x_{n}\right)_{n}$. For this hierarchy the following is proved.

THEOREM II. Let $\left(f_{n}\right)_{n}$ be a normalized weakly null sequence in $C\left(\omega^{\omega^{\xi}}\right)$. Then there exist $\zeta \leq \xi$ and $N \in[\mathbb{N}]$ such that for all $L \in[N]$ the sequence $\left(\zeta_{n}^{L}\right)_{n}$ is equivalent to the usual basis of $c_{0}$.

As follows from $[\mathrm{BP}]$, the spaces $C\left(\omega^{\omega^{\xi}}\right)$ determine all the isomorphs of $C(\mathcal{K})$ with $\mathcal{K}$ countable compact. The proof of the above theorem is deep and requires intricate arguments. This is naturally expected since for a sequence $\left(f_{n}\right)_{n}$ in $C(\mathcal{K})$ to be equivalent to the usual basis of $c_{0}$, a control of the global behavior of $\left(f_{n}\right)_{n}$ is required, which is not yet completely clarified. A positive solution to the above stated problem could also provide more understanding of the structure of $c_{0}$-sequences.

One of the goals of the present paper is to give an alternative proof of Theorem II in a slightly more general setting. In particular we introduce $c_{0}$-hierarchies and for such a hierarchy we prove the analog of the above theorem. Moreover some ingredients of the proof used to provide better upper estimates in $C\left(\omega^{\omega^{\xi}}\right)$ are of independent interest.

Let us pass to a presentation of the content of the paper. Throughout the paper we will use the representation of countable compact metric spaces by compact families of finite subsets of $\mathbb{N}$. In particular, the Schreier families $\left\{\mathcal{S}_{\xi}\right\}_{\xi<\omega_{1}}$ (see $\left.[\mathrm{AA}]\right)$ which correspond to the ordinals of the form $\omega^{\omega^{\xi}}$, $\xi<\omega_{1}$, play a key role in our proof.

In Section 2 we recall some well known results concerning mainly the compact families of finite subsets of $\mathbb{N}$. Section 3 is devoted to an improvement of Proposition 3.4 of [GOW]. This is one of the main results of the paper and states the following. 
Theorem A. Let $\gamma<\omega_{1}$ and $\left(f_{n}\right)_{n} \subseteq C\left(\mathcal{S}_{\gamma}\right)$ be a normalized weakly null sequence. Set

$$
\begin{aligned}
& \beta=\beta\left(\left(f_{n}\right)_{n}\right) \\
&=\min \{\xi \leq \gamma: \text { there exists } k \in \mathbb{N} \text { with } \varlimsup \\
&\left.\overline{\lim }\left\|\left.f_{n}\right|_{\mathcal{S}_{\gamma} \cap\left(\mathcal{S}_{\xi} * k\right)}\right\|>0\right\}
\end{aligned}
$$

and let $\delta<\omega_{1}$ be such that $\gamma=\beta+\delta$. Then there exists $M \in[\mathbb{N}], M=$ $\left\{m_{n}\right\}_{n}$, such that for every $\left(a_{n}\right)_{n} \in c_{00}(\mathbb{N})$,

$$
\left\|\sum a_{n} f_{m_{n}}\right\| \leq 5\left\|\sum a_{n} f_{m_{n}}\right\|_{\mathcal{S}_{\delta}} .
$$

In the above theorem $\mathcal{S}_{\xi} * k$ denotes the compact family containing the subsets of $\mathbb{N}$ which are unions of at most $k$ successive members of $\mathcal{S}_{\xi}$, and

$$
\left\|\sum a_{n} f_{m_{n}}\right\|_{\mathcal{S}_{\delta}}=\sup \left\{\left\|\sum_{n \in F} a_{n} f_{m_{n}}\right\|:\left\{m_{n}: n \in F\right\} \in \mathcal{S}_{\delta}\right\} .
$$

The aforementioned proposition in [GOW] provides an upper bound similar to (2) with the $\mathcal{S}_{\gamma}$ norm in place of $\mathcal{S}_{\delta}$. Let us observe here that if $\beta\left(\left(f_{n}\right)_{n}\right)=\gamma$ then $\delta=0$ and so Theorem $\mathrm{A}$ immediately gives that $\left(f_{n}\right)_{n}$ contains a subsequence equivalent to the usual basis of $c_{0}$. It is also worth pointing out that the set $\mathcal{S}_{\gamma} \cap\left(\mathcal{S}_{\xi} * k\right)$ which appears in the statement of the theorem does not correspond to a naturally defined subset of the ordinal $\omega^{\omega^{\gamma}}$ which is the ordinal analog of $\mathcal{S}_{\gamma}$. Moreover, the use of $\mathcal{S}_{\xi}$ instead of $\omega^{\omega^{\xi}}$ is important in the proof of the theorem. In particular, an ingredient is the quotient $\mathcal{F} / \mathcal{G}$ (with $\mathcal{F}, \mathcal{G}$ compact hereditary families) defined in this section. This is a new operation between families of finite subsets of $\mathbb{N}$ leading to another compact family and corresponds to an inverse of the convolution defined in $[\mathrm{AD}]$. Concerning the quotient $\mathcal{Q}=\left(\mathcal{S}_{\xi} * k\right) / \mathcal{S}_{\zeta}$ with $\zeta \leq \xi$ we show in the Appendix that the order of $\mathcal{Q}$ is equal to $\omega^{\delta} \cdot k$ where $\zeta+\delta=\xi$.

In Section 4 motivated by the normalized $\zeta$-averages introduced in [GOW] we define $c_{0}$-hierarchies. For a given normalized Schauder basic sequence $\left(x_{n}\right)_{n}$ a $c_{0}$-hierarchy is a family

$$
\mathcal{H}=\left\{\left(\zeta_{n}^{L}\right)_{n}: L \in[\mathbb{N}], \zeta<\omega_{1}\right\}
$$

of block subsequences of $\left(x_{n}\right)_{n}$ satisfying certain axioms. The basic example is what we call the "standard $c_{0}$-hierarchy", which is the family of normalized $\zeta$-averages of $[\mathrm{GOW}]$. It follows from the results of [GOW] that they satisfy the axioms.

In Section 5 we study properties of $c_{0}$-hierarchies in $C(\mathcal{K})$ spaces which are mostly of Ramsey type. Section 6 contains the main results for $c_{0^{-}}$ hierarchies. In particular, the following is shown, which extends the aforementioned Theorem II of [GOW].

Theorem B. Let $\mathcal{K}$ be a compact metric space, $\left(f_{n}\right)_{n} \subseteq C(\mathcal{K})$ be a normalized shrinking basic sequence and $\mathcal{H}$ be a $c_{0}$-hierarchy generated by 
$\left(f_{n}\right)_{n}$. Let $\gamma<\omega_{1}$ and $\mathcal{L}$ be a countable closed subset of $\mathcal{K}$ homeomorphic to a subset of $\omega^{\omega^{\gamma}}$. Suppose that for some $\zeta \geq \gamma$ and $N \in[\mathbb{N}]$, the family $\left\{\left(\left.\zeta_{n}^{L}\right|_{\mathcal{L}}\right)_{n}: L \in[N]\right\}$ is seminormalized. Then there exist $\beta \leq \gamma$ and $M \in[N]$ such that for all $L \in[M]$ the following hold:

(i) The block sequence $\left(\left.\beta_{n}^{L}\right|_{\mathcal{L}}\right)_{n}$ is equivalent the usual basis of $c_{0}$.

(ii) If $\zeta=\beta+\delta$ then $\left(\beta_{n}^{L}\right)_{n}$ is a $c_{0}^{\delta}$-spreading model.

The above theorem shows that $c_{0}$-hierarchies effectively determine a $c_{0^{-}}$ basis in spaces $C(\mathcal{K})$ with $\mathcal{K}$ a countable compact metric space and moreover for $\zeta>\gamma$ the second part of the conclusion of the theorem provides information on the global behavior of $\left(\beta_{n}^{L}\right)_{n}$ in arbitrary compact spaces $\mathcal{K}$. The proof of Theorem B proceeds by induction on the ordinal $\gamma$ and is based on Theorem A and the Ramsey type properties stated and proved in Section 5.

Let us describe briefly the main steps of the proof. First by the properties of $c_{0}$-hierarchies it turns out that it suffices to show only part (i) of the theorem for the special case of $\zeta=\gamma$. Identifying $\mathcal{L}$ with $\mathcal{S}_{\gamma}$ we set $\mathcal{L}_{\xi, k}=$ $\mathcal{S}_{\gamma} \cap\left(\mathcal{S}_{\xi} * k\right)$ for every ordinal $\xi \leq \gamma$ and $k \in \mathbb{N}$, and we define an analog of the index $\beta$ of Theorem A (see (1)) as follows:

$$
\begin{aligned}
\beta=\min \left\{\xi \leq \gamma: \exists N^{\prime} \in[N], \exists k \in \mathbb{N}\right. \text { such that } \\
\text { the set } \left.\left\{\left(\left.\gamma_{n}^{L}\right|_{\mathcal{L}_{\xi, k}}\right)_{n}: L \in\left[N^{\prime}\right]\right\} \text { is seminormalized }\right\} .
\end{aligned}
$$

Our aim is to prove that the above defined $\beta$ satisfies the conclusion of Theorem B. The case of $\beta=\gamma$ is treated easily. Indeed, in this case, by the properties of $c_{0}$-hierarchies we can find $N^{\prime} \in[N]$ such that $\beta\left(\left(\left.\gamma_{n}^{L}\right|_{\mathcal{L}}\right)_{n}\right)=$ $\gamma$ for all $L \in\left[N^{\prime}\right]$, and as we have already mentioned after Theorem A, this implies that for every $L \in\left[N^{\prime}\right]$ there exists a subsequence of $\left(\left.\gamma_{n}^{L}\right|_{\mathcal{L}}\right)_{n}$ equivalent to the usual basis of $c_{0}$. So the hard case is when $\beta<\gamma$. Here we use our inductive assumption and we conclude that there exist $N^{\prime} \in[N]$ and $k \in \mathbb{N}$ such that for all $L \in\left[N^{\prime}\right]$, the block sequence $\left(\beta_{n}^{L}\right)_{n}$ restricted to the closed subset $\mathcal{L}_{\beta, k}$ of $\mathcal{L}$ is equivalent to the usual basis of $c_{0}$, while on the other hand, $\left(\beta_{n}^{L}\right)_{n}$ is a $c_{0}^{\delta}$-spreading model for the whole space $\mathcal{K}$, where $\beta+\delta=\gamma$. Then applying again the properties of $c_{0}$-hierarchies and Theorem A we obtain the conclusion of Theorem B.

Finally, in Section 7 some consequences of the above theorem are also included.

\section{Preliminaries}

2.1. Notation. Let us first fix some notation. For an infinite subset $M$ of $\mathbb{N}$, by $[M]$ (resp. $[M]^{<\omega}$ ) we denote the set of all infinite (resp. finite) subsets of $M$. If $t$ is a finite subset of $\mathbb{N}$ then by $|t|$ we denote the cardinality of $t$. Also for $k \in \mathbb{N},[M] \leq k$ is the set of all finite subsets of $M$ of cardinality at most $k$. 
For $t_{1}, t_{2}$ finite subsets of $\mathbb{N}$ we write $t_{1}<t_{2}$ (resp. $t_{1} \leq t_{2}$ ) if either at least one of them is empty or $\max t_{1}<\min t_{2}\left(\operatorname{resp} . \max t_{1} \leq \min t_{2}\right)$. In particular if $n \in \mathbb{N}$ and $t_{1}=\{n\}$, we will write $n<t_{2}$ (resp. $n \leq t_{2}$ ) instead of $\{n\}<t_{2}$ (resp. $\{n\} \leq t_{2}$ ). Also the notation $t_{1} \preceq t_{2}$ means that both $t_{1}, t_{2}$ have the same cardinality and if they are nonempty then $t_{1}=\left\{n_{1}, \ldots, n_{k}\right\}$, $t_{2}=\left\{n_{1}^{\prime}, \ldots, n_{k}^{\prime}\right\}$ and $n_{i} \leq n_{i}^{\prime}$ for all $i=1, \ldots, k$. Finally, for $s, t$ finite subsets of $\mathbb{N}$, by $s \sqsubseteq t$ we denote that $s$ is an initial segment of $t$. If $s \sqsubseteq t$ and $s \neq t$ then we say that $s$ is a proper initial segment of $t$.

2.2. On families of finite subsets of $\mathbb{N}$. A family $\mathcal{F}$ of finite subsets of $\mathbb{N}$ is called compact if the set $\mathcal{X}_{\mathcal{F}}=\left\{1_{t}: t \in \mathcal{F}\right\}$ of characteristic functions of its members is a closed subset of $2^{\mathbb{N}}$; hereditary if for every $t \in \mathcal{F}, \mathcal{F}$ contains all subsets of $t$; and spreading if for every $t \in \mathcal{F}, \mathcal{F}$ contains all $t^{\prime} \subseteq \mathbb{N}$ with $t \preceq t^{\prime}$. If $\mathcal{F}$ has all the above three properties then $\mathcal{F}$ will be called a regular family.

If $\mathcal{F}$ is a compact family then identifying $\mathcal{F}$ with $\mathcal{X}_{\mathcal{F}}$, the CantorBendixson rank $|\mathcal{F}|_{\mathrm{CB}}$ is naturally defined. We define the order of a compact family $\mathcal{F}$, denoted by $o(\mathcal{F})$, to be the immediate predecessor of $|\mathcal{F}|_{\mathrm{CB}}$.

Observe that if $\mathcal{F}$ is assumed to be also hereditary then the first CantorBendixson derivative $\mathcal{F}^{\prime}$ of $\mathcal{F}$ is also a compact and hereditary family and

$$
\mathcal{F}^{\prime}=\{t \in \mathcal{F} \text { : there exists } L \in[\mathbb{N}] \text { such that } t \cup\{l\} \in \mathcal{F} \text { for all } l \in L\} \text {. }
$$

In particular if $\mathcal{F}$ is a regular family then $\mathcal{F}^{\prime}$ is also regular and in this case we have the following:

(i) $\mathcal{F}^{\prime}=\left\{t \in \mathcal{F}\right.$ : there exists $n_{0} \in \mathbb{N}$ such that $t \cup\{n\} \in \mathcal{F}$ for all $\left.n \geq n_{0}\right\}$.

(ii) The $\xi$-iterated Cantor-Bendixson derivative of $\mathcal{F}$ coincides with the $\xi$-iterated derivative of the well-founded tree $T_{\mathcal{F}}=(\mathcal{F}, \sqsubseteq)$ and therefore $o\left(T_{\mathcal{F}}\right)=o(\mathcal{F})+1$.

(iii) The set $\mathcal{F}_{\text {max }}$ of all $\subseteq$-maximal members of $\mathcal{F}$ coincides with the set of all $\sqsubseteq$-maximal members of $\mathcal{F}$.

(iv) $\mathcal{F}^{o(\mathcal{F})}=\{\emptyset\}$.

(v) For every $L \in[\mathbb{N}], o(\mathcal{F}[L])=o(\mathcal{F})$, where $\mathcal{F}[L]=\{t \in \mathcal{F}: t \subseteq L\}$.

Typical examples of regular families in $\mathbb{N}$ of transfinite order are the Schreier families $\mathcal{S}_{\xi}, \xi<\omega_{1}$ (see $[\mathrm{AO}]$ for $\xi<\omega$ and $[\mathrm{AA}]$ for general $\xi<\omega_{1}$ ).

Definition 1. For $\xi=0, \mathcal{S}_{0}=\{\{n\}: n \in \mathbb{N}\} \cup\{\emptyset\}$. If $\mathcal{S}_{\xi}$ has been defined then we set

$$
\mathcal{S}_{\xi+1}=\left\{\bigcup_{i=1}^{n} t_{i}: n \leq t_{1}<\cdots<t_{n}, t_{1}, \ldots, t_{n} \in \mathcal{S}_{\xi} \text { and } n \in \mathbb{N}\right\} .
$$

If $\xi$ is a limit ordinal then let $\left(\xi_{n}\right)_{n}$ be a strictly increasing sequence of 
ordinals with $\sup _{n} \xi_{n}=\xi$ and define

$$
\mathcal{S}_{\xi}=\left\{t \subseteq \mathbb{N} \text { : there exists } n \leq t \text { with } t \in \mathcal{S}_{\xi_{n}}\right\} .
$$

The following properties of the Schreier families are well known.

(i) For all $\xi<\omega_{1}, \mathcal{S}_{\xi}$ is a regular family of order $\omega^{\xi}$ and homeomorphic to the ordinal $\omega^{\omega^{\xi}}+1$.

(ii) For every $\xi<\zeta$, there exists $n_{0} \in \mathbb{N}$ such that $\left\{t \in \mathcal{S}_{\xi}: n_{0} \leq t\right\}$ $\subseteq \mathcal{S}_{\zeta}$

(iii) For every $\xi<\omega_{1}$ and every $L \in[\mathbb{N}]$ there exists a (unique) maximal member $t$ of $\mathcal{S}_{\xi}$ such that $t \sqsubseteq L$.

Given two compact and hereditary (resp. regular) families $\mathcal{F}, \mathcal{G}$ the convolution $\mathcal{F} * \mathcal{G}$ is defined as follows:

$$
\begin{aligned}
\mathcal{F} * \mathcal{G}=\left\{\bigcup_{i=1}^{n} t_{i}: \text { there exist } m_{1} \leq\right. & t_{1}<\cdots<m_{n} \leq t_{n} \\
& \text { with } \left.t_{1}, \ldots, t_{n} \in \mathcal{F},\left\{m_{i}\right\}_{i=1}^{n} \in \mathcal{G}\right\} .
\end{aligned}
$$

It is easy to see that the above operation is associative and that $\mathcal{F} * \mathcal{G}$ is a compact and hereditary (resp. regular) family.

In the following, for a family $\mathcal{F}$ and a $k \in \mathbb{N}$, we will write $\mathcal{F} * k$ to denote the family $\mathcal{F} *[\mathbb{N}]^{\leq k}$. Note that for all $\xi<\omega_{1}$,

$$
\mathcal{S}_{\xi+1}=\mathcal{S}_{\xi} * \mathcal{S}_{1} \text {. }
$$

The theory of families of finite subsets of $\mathbb{N}$ is closely related to Ramsey theory $[\mathrm{NW}],[\mathrm{PR}]$. The following is the fundamental principle of infinite combinatorics $[\mathrm{El}],[\mathrm{S}]$, which we will need almost throughout the paper.

A set $\mathcal{X} \subseteq[\mathbb{N}]$ is said to have the Ramsey property if for every $N \in[\mathbb{N}]$ there exists $M \in[N]$ such that either $[M]$ is contained in $\mathcal{X}$ or is disjoint from $\mathcal{X}$.

TheOREM 2. Every analytic subset of $[\mathbb{N}]$ has the Ramsey property.

Actually, we will need the above theorem only for open subsets of $[\mathbb{N}$ ] (for the Borel version of Theorem 2 see [GP]). One of the numerous consequences of this principle is the next result (see $[\mathrm{G}]$ ).

TheOREM 3. Let $\mathcal{F}, \mathcal{G}$ be hereditary families of finite subsets of $\mathbb{N}$. Then for every $N \in[\mathbb{N}]$ there exists $M \in[N]$ such that either $\mathcal{F}[M] \subseteq \mathcal{G}$ or $\mathcal{G}[M] \subseteq \mathcal{F}$

Notice that in particular if $\mathcal{F}, \mathcal{G}$ are regular and $o(\mathcal{F})<o(\mathcal{G})$ then the above theorem shows that for every $N \in[\mathbb{N}]$ there exists $M \in[N]$ such that $\mathcal{F}[M] \subseteq \mathcal{G}$.

For a detailed exposition of the results concerning families of finite subsets of $\mathbb{N}$ and applications in Ramsey and Banach space theory the reader 
is referred to $[\mathrm{T}]$. The survey paper by H. P. Rosenthal $[R]$ is an excellent source for results concerning $C(\mathcal{K})$ spaces.

3. Some upper estimates. Our primary aim here is to show that every seminormalized weakly null sequence in $C(\mathcal{K})$ with $\mathcal{K}$ a countable compact metric space contains a subsequence admitting certain upper estimates. Our results are refinements of the corresponding ones stated in [GOW]. In order to attain them we define two new notions. The first is the operation of the quotient of two families in $\mathbb{N}$ and the second is an ordinal index which we call the Schreier norming index of a seminormalized weakly null sequence of functions. Let us point out that the representation of countable compact metric spaces by families of finite subsets of $\mathbb{N}$ plays a key role in what follows.

3.1. Quotient families in $\mathbb{N}$. Let $\mathcal{G}$ be a family of finite subsets of $\mathbb{N}$ with the following properties:

(1) $\mathcal{G}$ is compact and hereditary.

(2) $\mathcal{G}$ covers $\mathbb{N}$, that is, $\mathbb{N}=\bigcup\{t: t \in \mathcal{G}\}$.

For each $L \in[\mathbb{N}]$, set $\mathcal{I}_{\mathcal{G}}(L)=\{t \in \mathcal{G}: t \sqsubseteq L\}$ and notice that $\mathcal{I}_{\mathcal{G}}(L)$ is a nonempty set containing at least the empty set and the singleton $\{\min L\}$. Furthermore, $\mathcal{I}_{\mathcal{G}}(L)$ is a finite subset of $\mathcal{G}$ and the partial orders $\sqsubseteq$ and $\subseteq$ coincide on $\mathcal{I}_{\mathcal{G}}(L)$.

Using the above remarks we find that every $L \in[\mathbb{N}]$ can be decomposed into a unique sequence $\left(t_{n}^{L}\right)_{n}$ of successive members of $\mathcal{G}$ defined by induction as follows. We let $t_{1}^{L}$ be the maximal (under inclusion or the initial segment ordering) member of $\mathcal{I}_{\mathcal{G}}(L)$. Suppose that $t_{1}^{L}<\cdots<t_{n}^{L}$ have been defined. Then we set $L_{n}=L \backslash \bigcup_{i=1}^{n} t_{i}^{L}$ and we define $t_{n+1}^{L}$ to be the maximal member of the set $\mathcal{I}_{\mathcal{G}}\left(L_{n}\right)$.

We call $\left(t_{n}^{L}\right)_{n}$ the $\mathcal{G}$-decomposition of $L$ and the set $q_{\mathcal{G}}(L)=\left\{\min t_{n}^{L}\right\}_{n}$ the $\mathcal{G}$-quotient of $L$.

For a given finite subset $s$ of $\mathbb{N}$ we set $N_{s}=s \cup\{n \in \mathbb{N}: n>\max s\}$ (if $s=\emptyset$ then $N_{\emptyset}=\mathbb{N}$ ) and we define the $\mathcal{G}$-quotient of $s$ to be the set $q_{\mathcal{G}}(s)=s \cap q_{\mathcal{G}}\left(N_{s}\right)$. It is easy to notice that for every $L \in[\mathbb{N}]$ with $s \sqsubseteq L$, we have $q_{\mathcal{G}}(s)=s \cap q_{\mathcal{G}}(L)$.

Definition 4. Let $\mathcal{F}, \mathcal{G}$ be compact and hereditary families of finite subsets of $\mathbb{N}$ and assume that $\mathcal{G}$ covers $\mathbb{N}$. Then the $\mathcal{G}$-quotient of $\mathcal{F}$, denoted by $\mathcal{F} / \mathcal{G}$, is defined to be the spreadification of the family of all $\mathcal{G}$-quotients of the members of $\mathcal{F}$, that is,

$$
\mathcal{F} / \mathcal{G}=\left\{t \subseteq \mathbb{N} \text { : there exists } s \in \mathcal{F} \text { such that } q_{\mathcal{G}}(s) \preceq t\right\} .
$$

Notice that $\mathcal{F} / \mathcal{G}$ is a regular family and if $\mathcal{F}$ is in addition spreading then 


$$
\mathcal{F} / \mathcal{G} \subseteq \mathcal{F}
$$

Also it is obvious that

$$
\mathcal{F} \subseteq \mathcal{G} *(\mathcal{F} / \mathcal{G})
$$

By (4) and (5) we see that for every regular family $\mathcal{F}$ and every compact and hereditary family $\mathcal{G}$ covering $\mathbb{N}$,

$$
o(\mathcal{F} / \mathcal{G}) \leq o(\mathcal{F}) \leq o(\mathcal{G}) \cdot o(\mathcal{F} / \mathcal{G})
$$

Let us point out here that it may happen that $o(\mathcal{F})=o(\mathcal{G})=o(\mathcal{F} / \mathcal{G})$ (see the Appendix).

Of particular importance will be the Schreier quotients $\left(\mathcal{S}_{\xi} * k\right) / \mathcal{S}_{\beta}$ for $\beta \leq \xi$ and $k \in \mathbb{N}$. The following result is proved in the Appendix.

Proposition 5. Let $\xi=\beta+\delta$ be countable ordinals, $k \in \mathbb{N}$ and $\mathcal{P}=$ $\left(\mathcal{S}_{\xi} * k\right) / \mathcal{S}_{\beta}$. Then $o(\mathcal{P})=\omega^{\delta} \cdot k$.

The above proposition and Theorem 3 yield the following.

Corollary 6. For every $N \in[\mathbb{N}]$ there exists $M \in[N]$ such that $\mathcal{P}[M] \subseteq \mathcal{S}_{\delta} *(k+1)$.

3.2. The Schreier norming index. Fix a compact and hereditary family $\mathcal{F}$ in $\mathbb{N}$ such that $\mathcal{F} \subseteq \mathcal{S}_{\gamma} * k$ for some $\gamma<\omega_{1}$ and $k \in \mathbb{N}$. Let also $\left(f_{n}\right)_{n}$ be a seminormalized weakly null sequence in $C(\mathcal{F})$.

Definition 7. We define $\beta\left(\left(f_{n}\right)_{n}\right)=\min \left\{\xi \leq \gamma:\right.$ there exists $l \in \mathbb{N}$ such that $\left.\varlimsup\left\|\left.f_{n}\right|_{\mathcal{F} \cap\left(\mathcal{S}_{\xi} * l\right)}\right\|>0\right\}$. The ordinal $\beta\left(\left(f_{n}\right)_{n}\right)$ will be called the Schreier norming index of the sequence $\left(f_{n}\right)_{n}$.

Notation. To each countable ordinal $\beta \geq 1$ we associate a sequence $\left(\beta_{i}\right)_{i}$ of ordinals as follows. In the case where $\beta$ is a successor ordinal, $\beta=$ $\beta^{\prime}+1$, we set $\beta_{i}=\beta^{\prime}$ for all $i \in \mathbb{N}$. If $\beta$ is a limit ordinal then $\left(\beta_{i}\right)_{i}$ is the strictly increasing sequence of ordinals converging to $\beta$ that was used in the definition of $\mathcal{S}_{\beta}$.

Concerning the above defined index we have the next proposition which is essentially a "sliding hump" argument.

Proposition 8. Let $\beta=\beta\left(\left(f_{n}\right)_{n}\right)$. Then given $N \in[\mathbb{N}]$ and a decreasing sequence $\left(\varepsilon_{n}\right)_{n}$ of positive scalars converging to zero there exist two strictly increasing sequences $\left(m_{n}\right)_{n=0}^{\infty}$ and $\left(l_{n}\right)_{n=0}^{\infty}$ such that $m_{0}=l_{0}=1$ and for every $n \geq 1$ the following are satisfied:

(a) $m_{n} \in N$.

(b) $l_{n-1}<m_{n}<l_{n}$.

(c) For every $t \in \mathcal{F}$ with $t \subseteq\left[1, l_{n-1}\right),\left|f_{m_{n}}(t)\right|<\varepsilon_{n} / 2$. 
(d) If $\beta \geq 1$ and $\left(\beta_{i}\right)_{i}$ is the sequence of ordinals associated to $\beta$, then for all $i \leq l_{n-1}$,

$$
\left\|f_{m_{n}} \mid \mathcal{F} \cap\left(\mathcal{S}_{\beta_{i}} * 2 l_{n-1}\right)\right\|<\varepsilon_{n} / 2 .
$$

(e) For every $t, t^{\prime} \in \mathcal{F}$ with $t \cap\left[1, l_{n}\right)=t^{\prime} \cap\left[1, l_{n}\right)$,

$$
\left|f_{m_{n}}(t)-f_{m_{n}}\left(t^{\prime}\right)\right|<\varepsilon_{n} / 2 \text {. }
$$

Proof. The sequences $\left(m_{n}\right)_{n},\left(l_{n}\right)_{n}$ are constructed by induction. For $n=0$, we set $m_{0}=l_{0}=1$. Suppose that for some $n \in \mathbb{N}$ and every $i=0, \ldots, n-1, m_{i}$ and $l_{i}$ have been chosen so as to satisfy the conditions of the proposition. Since $\left(f_{m}\right)_{m}$ is weakly null there is an $n_{0} \in \mathbb{N}$ such that $\left|f_{m}(t)\right|<\varepsilon_{n} / 2$ for all $t \subseteq\left[1, l_{n-1}\right), t \in \mathcal{F}$ and all $m \geq n_{0}$. Moreover, if $\beta \geq 1$ then notice that $\lim _{m}\left\|\left.f_{m}\right|_{\mathcal{F} \cap\left(\mathcal{S}_{\beta_{i}} * 2 l_{n-1}\right)}\right\|=0$ for all $i \leq l_{n-1}$, by Definition 7. Therefore there exists $n_{1} \in \mathbb{N}$ such that for all $m \geq n_{1}$ and $i \leq l_{n-1}$ we have $\left\|\left.f_{m}\right|_{\mathcal{F} \cap\left(\mathcal{S}_{\beta_{i}} * 2 l_{n-1}\right)}\right\|<\varepsilon_{n} / 2$. Hence we can clearly choose $m_{n}>l_{n-1}$ satisfying (c), and if $\beta \geq 1$, condition (d) as well. Finally, by the uniform continuity of $f_{m_{n}}$, there exists $l_{n}>m_{n}$ satisfying condition (e).

3.3. The main results. Let us start with the following definition extending the one of Schreier spaces in $[\mathrm{AA}]$.

Definition 9. Let $X$ be a Banach space, $\left(x_{n}\right)_{n}$ be a basic sequence in $X$ and $\mathcal{A}$ be a hereditary family of subsets of $\mathbb{N}$. For every $\left(a_{n}\right)_{n} \in c_{00}(\mathbb{N})$ we set

$$
\left\|\sum_{n} a_{n} x_{n}\right\|_{\mathcal{A}}=\sup \left\{\left\|\sum_{n \in F} a_{n} x_{n}\right\|: F \in \mathcal{A}\right\} .
$$

The quantity $\|\cdot\|_{\mathcal{A}}$ is in general a seminorm on the linear span of $\left(x_{n}\right)_{n}$ unless the family $\mathcal{A}$ covers $\mathbb{N}$. In general notice that for an $M \in[\mathbb{N}], M=$ $\left(m_{n}\right)_{n}$, we have

$$
\left\|\sum_{n} a_{n} x_{m_{n}}\right\|_{\mathcal{A}}=\sup \left\{\left\|\sum_{n \in F} a_{n} x_{m_{n}}\right\|: F \subseteq \mathbb{N} \text { and }\left\{m_{n}: n \in F\right\} \in \mathcal{A}\right\}
$$

and if $\mathcal{A}$ covers $M$ then $\left(x_{m_{n}}\right)_{n}$ under this norm is an 1-unconditional basic sequence.

THEOREM 10. Let $\gamma<\omega_{1}$ and $\mathcal{F}$ be a compact and hereditary family of finite subsets of $\mathbb{N}$ such that $\mathcal{F} \subseteq \mathcal{S}_{\gamma} * k$ for some $k \in \mathbb{N}$. Let $\left(f_{n}\right)_{n}$ be a seminormalized weakly null sequence in $C(\mathcal{F})$ and $\beta=\beta\left(\left(f_{n}\right)_{n}\right)$ be the Schreier norming index of $\left(f_{n}\right)_{n}$. Set

$$
\mathcal{Q}=\mathcal{F} / \mathcal{S}_{\beta}
$$

and let $\delta$ be the unique ordinal so that

$$
\beta+\delta=\gamma
$$


Then given $0<\varepsilon<1$, there exists $M \in[\mathbb{N}]$ covered by $\mathcal{Q}, M=\left(m_{n}\right)_{n}$, such that $\left(f_{m_{n}}\right)_{n}$ is a basic sequence in $C(\mathcal{F})$ and for every $\left(a_{n}\right)_{n} \in c_{00}(\mathbb{N})$, the following is satisfied:

$$
\left\|\sum_{n} a_{n} f_{m_{n}}\right\| \leq \frac{2}{1-\varepsilon}\left\|\sum_{n} a_{n} f_{m_{n}}\right\|_{\mathcal{Q}} \leq \frac{2 k+2}{1-\varepsilon}\left\|\sum_{n} a_{n} f_{m_{n}}\right\|_{\mathcal{S}_{\delta}} .
$$

REMARK 1. If $\mathcal{F}$ is regular then $\mathcal{Q} \subseteq \mathcal{F}$ and so by (7) we obtain the upper estimate of Proposition 3.4 in [GOW]:

$$
\left\|\sum_{n} a_{n} f_{m_{n}}\right\| \leq \frac{2}{1-\varepsilon}\left\|\sum_{n} a_{n} f_{m_{n}}\right\|_{\mathcal{F}}
$$

As mentioned in [GOW], an upper bound similar to (8) has also been obtained in $[\mathrm{L}]$.

Proof. Let $c>0$ be such that $\inf _{n}\left\|f_{n}\right\| \geq c$ and let $\left(\varepsilon_{n}\right)_{n}$ be a sequence of positive scalars such that $\sum_{n} \varepsilon_{n}<\varepsilon c / 4$. Clearly $\mathcal{F}$ contains infinitely many members and so we can choose $N \in[\mathbb{N}]$ such that $N$ is covered by $\mathcal{F}$. We may also assume that $\left(f_{n}\right)_{n \in N}$ is a 2-basic sequence.

Let $\left(m_{n}\right)_{n=0}^{\infty},\left(l_{n}\right)_{n=0}^{\infty}$ be the sequences from Proposition 8 . We will show that $M=\left(m_{n}\right)_{n=1}^{\infty}$ satisfies the conclusion of the theorem. For every $t \in \mathcal{F}$ we set

$$
F_{t}=\left\{n \in \mathbb{N}:\left|f_{m_{n}}(t)\right| \geq \varepsilon_{n}\right\} \quad \text { and } \quad G_{t}=\left\{m_{n}: n \in F_{t}\right\} .
$$

It is easy to verify that for every $\left(a_{n}\right)_{n} \in c_{00}(\mathbb{N})$,

$$
\left\|\sum_{n} a_{n} f_{m_{n}}\right\| \leq \frac{1}{1-\varepsilon} \sup \left\{\left|\sum_{n \in F_{t}} a_{n} f_{m_{n}}(t)\right|: t \in \mathcal{F}\right\} .
$$

For every $n \in \mathbb{N}$ and every $t \in \mathcal{F}$ set

$$
t_{n}=t \cap\left[l_{n-1}, l_{n}\right) .
$$

Claim 1. For every $t \in \mathcal{F}$ and every $n \in F_{t}$ the set $t_{n}$ is a nonempty member of $\mathcal{F}$. In particular if $\beta \geq 1$ and $\left(\beta_{i}\right)_{i}$ is the sequence associated to $\beta$ then $t_{n} \notin \mathcal{S}_{\beta_{i}} * l_{n-1}$ for all $i \leq l_{n-1}$.

Proof. Fix a $t \in \mathcal{F}$ and an $n \in F_{t}$. Since $\mathcal{F}$ is hereditary it is clear that $t_{n}$ belongs to $\mathcal{F}$. Set $t^{\prime}=t \cap\left[1, l_{n-1}\right)$. By part (c) of Proposition 8 we get

$$
\left|f_{m_{n}}\left(t^{\prime}\right)\right|<\varepsilon_{n} / 2
$$

and by part (e) of the same proposition we conclude that

$$
\left|f_{m_{n}}\left(t^{\prime} \cup t_{n}\right)-f_{m_{n}}(t)\right|<\varepsilon_{n} / 2 .
$$

Suppose that $t_{n}=\emptyset$. Then $t^{\prime}=t^{\prime} \cup t_{n}$ and by (10) and (11) we infer that $\left|f_{m_{n}}(t)\right|<\varepsilon_{n}$, which is a contradiction since $n \in F_{t}$. Therefore $t_{n} \neq \emptyset$.

Let $\beta \geq 1$ and assume that $t_{n} \in \mathcal{S}_{\beta_{i}} * l_{n-1}$ for some $i \leq l_{n-1}$. Since $\left|t^{\prime}\right|<l_{n-1}$ we have $t^{\prime} \in \mathcal{S}_{\beta_{i}} * l_{n-1}$. Hence $t^{\prime} \cup t_{n} \in \mathcal{S}_{\beta_{i}} * 2 l_{n-1}$ and therefore 
by Proposition $8(\mathrm{~d})$ we get

$$
\left|f_{m_{n}}\left(t^{\prime} \cup t_{n}\right)\right|<\varepsilon_{n} / 2 .
$$

By (11) and (12) we find that again $\left|f_{m_{n}}(t)\right|<\varepsilon_{n}$, which is a contradiction.

Claim 2. Suppose that $\beta \geq 1$ and let $t \in \mathcal{F}$ and $n \in F_{t}$. Then for every $1 \leq l \leq l_{n-1}$ there exists a maximal element $s$ of $\mathcal{S}_{\beta}$ such that $s$ is a proper initial segment of $\{l\} \cup t_{n}$.

Proof. Assume that the conclusion is false. Then by the stability properties of the Schreier families we obtain $\{l\} \cup t_{n} \in \mathcal{S}_{\beta}$.

Suppose first that $\beta$ is a successor ordinal and let $\beta=\beta^{\prime}+1$. Since $l \leq l_{n-1}$, we have $\{l\} \cup t_{n} \in \mathcal{S}_{\beta^{\prime}} * l_{n-1}$. But then $t_{n} \in \mathcal{S}_{\beta^{\prime}} * l_{n-1}$, which contradicts Claim 1.

On the other hand, if $\beta$ is a limit ordinal then there is an $i \leq l_{n-1}$ such that $\{l\} \cup t_{n} \in \mathcal{S}_{\beta_{i}}$ and therefore $t_{n} \in \mathcal{S}_{\beta_{i}}$, which is again a contradiction.

Claim 3. For every $t \in \mathcal{F}$ with $G_{t} \neq \emptyset, G_{t} \backslash\left\{\min G_{t}\right\} \in \mathcal{Q}$.

Proof. Fix $t \in \mathcal{F}$ with $G_{t} \neq \emptyset$. By Claim 1 , we have $F_{t} \subseteq\{n \in \mathbb{N}$ : $\left.t_{n} \neq \emptyset\right\}$ and therefore $F_{t}$ is a finite subset of $\mathbb{N}$. Set

$$
F_{t}=\left\{n_{1}<\cdots<n_{d}\right\} \text { and } G_{t}=\left\{m_{n_{1}}<\cdots<m_{n_{d}}\right\} .
$$

Suppose first that $\beta=0$. For each $1 \leq i<d$, we set $m_{n_{i}}^{\prime}=\min t_{n_{i}}$. Then observe that $m_{n_{i}}^{\prime}<m_{n_{i+1}}$ for all $1 \leq i<d$ and therefore $\left\{m_{n_{i}}^{\prime}: 1 \leq i<d\right\} \preceq$ $G_{t} \backslash\left\{\min G_{t}\right\}$. Since $\left\{m_{n_{i}}^{\prime}: 1 \leq i<d\right\} \in \mathcal{F}$ and $\mathcal{Q}=\mathcal{F} / \mathcal{S}_{0}$ is clearly the spreadification of $\mathcal{F}$, the conclusion of the claim follows.

Assume now that $\beta \geq 1$. Applying successively Claim 2 we can choose maximal members $s_{1}<\cdots<s_{d-1}$ of $\mathcal{S}_{\beta}$ such that for every $1 \leq i<d$, $\min s_{i} \in t_{n_{i}}$ and $s_{i} \backslash\left\{\min s_{i}\right\}$ is a proper initial segment of $t_{n_{i+1}}$. Setting $s=$ $\bigcup_{1 \leq i<d} s_{i}$ we find that $q_{\mathcal{S}_{\beta}}(s)=\left\{\min s_{i}: 1 \leq i<d\right\}$. Since min $s_{i}<m_{n_{i+1}}$ for each $1 \leq i<d$, we have $q_{\mathcal{S}_{\beta}}(s) \preceq G_{t} \backslash\left\{\min G_{t}\right\}$, and as $s \in \mathcal{F}$, it follows that $G_{t} \backslash\left\{\min G_{t}\right\} \in \mathcal{Q}$.

Since $\mathcal{F}$ covers $N$ and $M \in[N]$ we see that $\mathcal{Q}$ covers $M=\left\{m_{n}: n \in \mathbb{N}\right\}$ and so by Claim 3 we have $G_{t} \in \mathcal{Q} * 2$ for all $t \in \mathcal{F}$. Therefore for every $\left(a_{n}\right)_{n} \in c_{00}(\mathbb{N})$, and every $t \in \mathcal{F}$,

$$
\left|\sum_{n \in F_{t}} a_{n} f_{m_{n}}(t)\right| \leq 2\left\|\sum a_{n} f_{m_{n}}\right\|_{\mathcal{Q}} .
$$

By (9) and (13) we obtain

$$
\left\|\sum_{n} a_{n} f_{m_{n}}\right\| \leq \frac{2}{1-\varepsilon}\left\|\sum_{n} a_{n} f_{m_{n}}\right\|_{\mathcal{Q}}
$$

To complete the proof let $\mathcal{P}=\left(\mathcal{S}_{\gamma} * k\right) / \mathcal{S}_{\beta}$. Then $\mathcal{Q} \subseteq \mathcal{P}$ and by Corollary 6 , we may assume that $\mathcal{Q}[M] \subseteq \mathcal{P}[M] \subseteq \mathcal{S}_{\delta} *(k+1)$. Hence every finite subset 
of $M$ which belongs to $\mathcal{Q}$ is the union of at most $k+1$ members of $\mathcal{S}_{\delta}$ and therefore

$$
\left\|\sum_{n} a_{n} f_{m_{n}}\right\|_{\mathcal{Q}} \leq \frac{2 k+2}{1-\varepsilon}\left\|\sum_{n} a_{n} f_{m_{n}}\right\|_{\mathcal{S}_{\delta}} .
$$

By (14) and (15) the proof of the theorem is complete.

Corollary 11. Let $\gamma<\omega_{1}$ and $\mathcal{F}$ be a compact and hereditary family of finite subsets of $\mathbb{N}$ such that $\mathcal{F} \subseteq \mathcal{S}_{\gamma} * k$ for some $k \in \mathbb{N}$. Let $\left(f_{n}\right)_{n}$ be a seminormalized weakly null sequence in $C(\mathcal{F})$ and $\beta\left(\left(f_{n}\right)\right)$ be the Schreier norming index of $\left(f_{n}\right)_{n}$. If $\beta\left(\left(f_{n}\right)_{n}\right)=\gamma$, then $\left(f_{n}\right)_{n}$ contains a subsequence equivalent to the usual basis of $c_{0}$.

Proof. Since $\beta\left(\left(f_{n}\right)_{n}\right)=\gamma$ we see that $\delta=0$. Hence Theorem 10 shows that there exists a subsequence $\left(f_{m_{n}}\right)_{n}$ of $\left(f_{n}\right)_{n}$ which is a basic sequence and a constant $C>0$ such that $\left\|\sum_{n} a_{n} f_{m_{n}}\right\| \leq C \max _{n}\left|a_{n}\right|$ for every $\left(a_{n}\right)_{n} \in c_{00}(\mathbb{N})$. Therefore $\left(f_{m_{n}}\right)_{n}$ is equivalent to the usual basis of $c_{0}$.

Before we continue with the presentation of our results let us recall the definition of the $c_{0}^{\delta}\left(\right.$ resp. $\left.l_{1}^{\delta}\right)$-spreading models.

Definition 12. Let $\left(x_{n}\right)_{n}$ be a basic sequence in a Banach space $X$ and let $\delta$ be a countable ordinal. Then a seminormalized block subsequence $\left(z_{n}\right)_{n}$ of $\left(x_{n}\right)_{n}$ is called a $c_{0}^{\delta}$ (resp. an $l_{1}^{\delta}$ )-spreading model if there is a constant $C>0$ such that

$$
\left\|\sum_{i \in F} a_{i} z_{i}\right\| \leq C \max _{i \in F}\left|a_{i}\right| \quad\left(\text { resp. }\left\|\sum_{i \in F} a_{i} z_{i}\right\| \geq C \sum_{i \in F}\left|a_{i}\right|\right)
$$

for every finite sequence $\left(a_{i}\right)_{i \in F}$ of scalars and every finite subset $F$ of $\mathbb{N}$ such that $\left\{\min \operatorname{supp} z_{i}: i \in F\right\}$ is a member of $\mathcal{S}_{\delta}$.

The next result is an extension of Corollary 11; it follows easily from Theorem 10.

COROLlary 13. Let $\gamma<\omega_{1}$ and $\mathcal{F}$ be a compact and hereditary family of finite subsets of $\mathbb{N}$ such that $\mathcal{F} \subseteq \mathcal{S}_{\gamma} * k$ for some $k \in \mathbb{N}$. Let $\left(f_{n}\right)_{n}$ be a seminormalized weakly null sequence in $C(\mathcal{F})$ and $\beta=\beta\left(\left(f_{n}\right)_{n}\right)$ be the Schreier norming index of $\left(f_{n}\right)_{n}$. If $\left(f_{n}\right)_{n}$ is a $c_{0}^{\delta}$-spreading model where $\beta+\delta=\gamma$ then $\left(f_{n}\right)_{n}$ contains a subsequence equivalent to the usual basis of $c_{0}$.

CoROllary 14. Let $\gamma<\omega_{1}$ and $\mathcal{F}$ be a compact and hereditary family of finite subsets of $\mathbb{N}$ such that $\mathcal{F} \subseteq \mathcal{S}_{\gamma} * k$ for some $k \in \mathbb{N}$. Let $\left(f_{n}\right)_{n}$ be a seminormalized weakly null sequence in $C(\mathcal{F})$ and $\beta=\beta\left(\left(f_{n}\right)_{n}\right)$ be the Schreier norming index of $\left(f_{n}\right)_{n}$. Let $l_{0} \in \mathbb{N}$ be such that $\varlimsup\left\|\left.f_{n}\right|_{\mathcal{F} \cap\left(\mathcal{S}_{\beta} * l_{0}\right)}\right\|$ $>0$. Then there exists a subsequence $\left(g_{n}\right)_{n}$ of $\left(f_{n}\right)_{n}$ such that if $\mathcal{G}=\mathcal{F} \cap$ $\left(\mathcal{S}_{\beta} * l_{0}\right)$ then $\left(\left.g_{n}\right|_{\mathcal{G}}\right)_{n}$ is equivalent to the usual basis of $c_{0}$. 
Proof. For each $n \in \mathbb{N}$, set $h_{n}=\left.f_{n}\right|_{\mathcal{G}}$. Without loss of generality we may assume that $\left(h_{n}\right)_{n}$ is seminormalized. Since $\mathcal{G}$ is a compact and hereditary family in $\mathbb{N}$ and $\mathcal{G} \subseteq \mathcal{S}_{\beta} * l_{0}$ the Schreier norming index $\beta\left(\left(h_{n}\right)_{n}\right)$ is defined and $\beta\left(\left(h_{n}\right)_{n}\right) \leq \beta$. By Corollary 13, it suffices to show that $\beta\left(\left(h_{n}\right)_{n}\right)=\beta$. Indeed, choose $\xi<\beta$ and $l \in \mathbb{N}$. Then $\mathcal{G} \cap\left(\mathcal{S}_{\xi} * l\right) \subseteq \mathcal{F} \cap\left(\mathcal{S}_{\xi} * l\right)$ and therefore

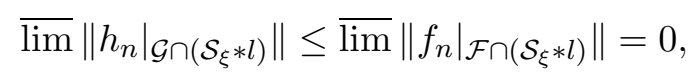

which yields our claim.

4. $c_{0}$-Hierarchies generated by basic sequences. In this section we proceed to the definition of $c_{0}$-hierarchies generated by a normalized basic sequence $\vec{x}=\left(x_{n}\right)_{n}$. The three axioms that any such hierarchy must fulfill have been extracted from the fundamental properties of the hierarchy of normalized averages defined in [GOW]. However, as examples indicate, there are many other hierarchies which could appear useful in applications. Moreover, the present approach gives a more transparent access to the proofs of the final results.

Let $\vec{x}=\left(x_{n}\right)_{n}$ be a normalized basic sequence in a Banach space $X$. With every countable ordinal and every $L \in[\mathbb{N}]$ we associate a block subsequence of $\left(x_{n}\right)_{n}$ denoted by $\left(\zeta_{n}^{L}\right)_{n}$. The collection

$$
\mathcal{H}=\left\{\left(\zeta_{n}^{L}\right)_{n}: \zeta<\omega_{1}, L \in[\mathbb{N}]\right\}
$$

of all these sequences will be called a $c_{0}$-hierarchy generated by $\vec{x}$ if it satisfies the following axioms.

Axiom 1. There exist constants $0<C_{1} \leq C_{2}$ such that for all $\zeta<\omega_{1}$, $L \in[\mathbb{N}]$ and $n \in \mathbb{N}$,

$$
C_{1} \leq\left\|\zeta_{n}^{L}\right\| \leq C_{2}
$$

A block of $\left(x_{n}\right)_{n}$ of the form $\zeta_{n}^{L}$ will be called a $\zeta$-block.

Axiom 2 (Ramsey axiom). For each $\zeta<\omega_{1}$ the following are satisfied.

(i) For every $L \in[\mathbb{N}], \bigcup_{n} \operatorname{supp} \zeta_{n}^{L}=L$.

(ii) For every $\zeta$-block $u$ and every $L \in[\mathbb{N}]$ such that $\operatorname{supp} u \sqsubseteq L, \zeta_{1}^{L}=u$.

As we will see in the next section Axiom 2 will provide certain strong Ramsey properties of the $\zeta$-blocks.

Notation. Let $\zeta<\omega_{1}$ and $N \in[\mathbb{N}]$. A $\zeta$-block $u$ is said to be supported by $N$ if $\operatorname{supp} u \subseteq N$. We set

$$
\mathcal{A}^{\zeta, N}=\{\operatorname{supp} u: u \text { is a } \zeta \text {-block supported by } N\}
$$

and let $\mathcal{B}^{\zeta, N}$ be the downward closure of $\mathcal{A}^{\zeta, N}$ relative to inclusion, that is, $\mathcal{B}^{\zeta, N}=\left\{t \subseteq \mathbb{N}\right.$ : there exists $s \in \mathcal{A}^{\zeta, N}$ such that $\left.t \subseteq s\right\}$. 
It is clear that for $N^{\prime}, N \in[\mathbb{N}]$, if $N^{\prime} \subseteq N$ then for all $\zeta<\omega_{1}$,

$$
\mathcal{A}^{\zeta, N^{\prime}} \subseteq \mathcal{A}^{\zeta, N} \quad \text { and } \quad \mathcal{B}^{\zeta, N^{\prime}} \subseteq \mathcal{B}^{\zeta, N} .
$$

Proposition 15. For every $\zeta<\omega_{1}$ and every $N \in[\mathbb{N}]$, the family $\mathcal{B}^{\zeta, N}$ is a compact family of finite subsets of $\mathbb{N}$.

Proof. Let $\zeta<\omega_{1}$. By (16) it suffices to show the assertion for $N=\mathbb{N}$. Assume on the contrary that $\mathcal{B}^{\zeta, \mathbb{N}}$ is not compact. Then since it is a hereditary family there exists an infinite subset $M$ of $\mathbb{N}$ such that every finite subset of $M$ belongs to $\mathcal{B}^{\zeta, \mathbb{N}}$. Let $t_{1} \sqsubset t_{2} \sqsubset \cdots$ be a strictly increasing sequence of initial segments of $M$. Then there exists a sequence $\left(s_{n}\right)_{n}$ of members of $\mathcal{A}^{\zeta, \mathbb{N}}$ such that $t_{n} \subseteq s_{n}$ for all $n \in \mathbb{N}$. By Axiom 2(ii), for each $n$ we can choose $L_{n} \in[\mathbb{N}]$ such that $s_{n}=\operatorname{supp} \zeta_{1}^{L_{n}}$. By passing to a subsequence we may assume that $\left(L_{n}\right)_{n}$ converges to a subset $L$ of $\mathbb{N}$. Observe that $t_{k} \subseteq L_{n}$ for every $k \leq n$, and therefore $M \subseteq L$. In particular $L$ is an infinite subset of $\mathbb{N}$. Set $s=\operatorname{supp} \zeta_{1}^{L}$. Then by Axiom 2(i), $s \sqsubseteq L$ and so there exists $n_{0}$ such that $s \sqsubseteq L_{n}$ for all $n \geq n_{0}$. Hence by Axiom 2(ii), for all $n \geq n_{0}, \zeta_{1}^{L_{n}}=\zeta_{1}^{L}$ and so $s_{n}=s$. But then $t_{n} \subseteq s$ for all $n$, which is impossible since $\left(t_{n}\right)_{n}$ is strictly increasing.

REMARK 2. The above proposition is actually a consequence of the fact that $\mathcal{A}^{\zeta, \mathbb{N}}$ is a maximal thin family of finite subsets of $\mathbb{N}$. Recall that a family $\mathcal{F}$ is called thin if for every pair $s, t$ of distinct members of $\mathcal{F}, s \nsubseteq t$ (this definition is due to Nash-Williams [NW]; for transfinite analogs see [PR]). We refer the reader to the survey paper by $\mathrm{S}$. Todorcevic $[\mathrm{T}]$ for a detailed exposition of related results. Observe also that Axiom 2 implies that the family of all $\zeta$-blocks is countable as it is in one-to-one correspondence to $\mathcal{A}^{\zeta, \mathbb{N}}$.

Terminology. Let $\zeta<\omega_{1}$ and $N \in[\mathbb{N}]$. Then by a $(\zeta,[N])$-sequence we will mean every block subsequence of $\left(x_{n}\right)_{n}$ of the form $\left(\zeta_{n}^{L}\right)_{n}$ with $L \in[N]$.

By Axiom 2 and by induction we easily obtain the following. For every $n \in \mathbb{N}$, every block sequence $u_{1}<\cdots<u_{n}$ of $\zeta$-blocks and every $L \in[\mathbb{N}]$ such that $\bigcup_{i=1}^{n} \operatorname{supp} u_{i}$ is an initial segment of $L$, we have $\zeta_{i}^{L}=u_{i}$, for all $i=1, \ldots, n$. Hence if $\left(u_{n}\right)_{n}$ is a block subsequence of $\left(x_{n}\right)_{n}$ such that every $u_{n}$ is a $\zeta$-block then setting $L=\bigcup_{n} \operatorname{supp} u_{n}$, we have $\zeta_{n}^{L}=u_{n}$ for all $n \in \mathbb{N}$. In particular, every subsequence of a $(\zeta,[N])$-sequence is also a $(\zeta,[N])$ sequence. More precisely, if $\left(m_{n}\right)_{n}$ is a strictly increasing sequence of natural numbers then for all $L \in[\mathbb{N}]$ and $n, \zeta_{m_{n}}^{L}=\zeta_{n}^{L^{\prime}}$ where $L^{\prime}=\bigcup_{n} \operatorname{supp} \zeta_{m_{n}}^{L}$.

Definition 16. Let $\beta<\omega_{1}$ and $\varepsilon>0$. A block $u$ of $\left(x_{n}\right)_{n}$ will be said to admit an $(\varepsilon, \beta)$-analysis if there exist $\beta$-blocks $b_{1}<\cdots<b_{n}$ and scalars $\lambda_{1}, \ldots, \lambda_{n}$ such that the following are satisfied:

(i) $\bigcup_{i=1}^{n} \operatorname{supp} b_{i} \subseteq \operatorname{supp} u$. 
(ii) $\left\|u-\sum_{i=1}^{n} \lambda_{i} b_{i}\right\|<\varepsilon$.

(iii) ||$\lambda_{i}|-| \lambda_{j}||<\varepsilon$ for all $i, j \in\{1, \ldots, n\}$.

The quantity $\max \left\{\left|\lambda_{i}\right|: i=1, \ldots, n\right\}$ will be called the weight of the $(\varepsilon, \beta)$ analysis of $u$. Also every subset of the set $\left\{\min \operatorname{supp} b_{i}: i=1, \ldots, n\right\}$ will be called an $(\varepsilon, \beta)$-admissible set resulting from $u$.

Notation. Given $\varepsilon>0, \beta<\zeta<\omega_{1}$ and $N \in[\mathbb{N}]$, we let $\mathcal{A}_{\varepsilon, \beta}^{\zeta, N}$ be the family of all $(\varepsilon, \beta)$-admissible sets resulting from $\zeta$-blocks supported by $N$. It is clear that $\mathcal{A}_{\varepsilon, \beta}^{\zeta, N}$ is a hereditary family and

$$
\mathcal{A}_{\varepsilon, \beta}^{\zeta, N} \subseteq \mathcal{B}^{\zeta, N} .
$$

Also for $N^{\prime}, N \in[\mathbb{N}]$, if $N^{\prime} \subseteq N$ then

$$
\mathcal{A}_{\varepsilon, \beta}^{\zeta, N^{\prime}} \subseteq \mathcal{A}_{\varepsilon, \beta}^{\zeta, N} .
$$

By (17) and Proposition 15 we immediately obtain the following.

Proposition 17. For all $\varepsilon>0, \beta<\zeta<\omega_{1}$ and $N \in[\mathbb{N}], \mathcal{A}_{\varepsilon, \beta}^{\zeta, N}$ is a compact family of finite subsets of $\mathbb{N}$.

Axiom 3 (Reduction axiom). Let $\beta<\zeta$ be countable ordinals and let $\delta \geq 1$ be the unique ordinal so that $\beta+\delta=\zeta$. Then for every $\varepsilon>0$ and every $N \in[\mathbb{N}]$ the following are satisfied:

(i) The family $\mathcal{A}_{\varepsilon, \beta}^{\zeta, N}$ of all $(\varepsilon, \beta)$-admissible sets resulting from $\zeta$-blocks supported by $N$ has order at least $\omega^{\delta}$.

(ii) There exists $M \in[N]$ such that every $\zeta$-block supported by $M$ admits an $(\varepsilon, \beta)$-analysis.

Remark 3. Let $\zeta<\omega_{1}$ and $N \in[\mathbb{N}]$. Notice that by Axiom 2(i) we simply had $o\left(\mathcal{B}^{\zeta, N}\right) \geq 1$. But by Axiom 3(i) (setting $\beta=0$ ) and (17) we now have

$$
o\left(\mathcal{B}^{\zeta, N}\right) \geq \omega^{\zeta} .
$$

Therefore for every $\zeta<\omega_{1}$ and $N \in[\mathbb{N}]$ the complexity of the $\zeta$-blocks supported by $N$ is strictly increasing.

Proposition 18. Let $\zeta=\beta+\delta, \delta \geq 1$, be countable ordinals, $N \in[\mathbb{N}]$ and $\varepsilon>0$. Then there exists $L \in[N]$ such that $F \backslash\{\min F\} \in \mathcal{A}_{\varepsilon, \beta}^{\zeta, N}$ for every $F \in \mathcal{S}_{\delta}[L]$.

Proof. First notice that for every $L \in[N]$,

$$
\mathcal{A}_{\varepsilon, \beta}^{\zeta, L} \subseteq \mathcal{A}_{\varepsilon, \beta}^{\zeta, N}[L] .
$$


Indeed, by (18), $\mathcal{A}_{\varepsilon, \beta}^{\zeta, L} \subseteq \mathcal{A}_{\varepsilon, \beta}^{\zeta, N} \cap[L]^{<\omega}=\mathcal{A}_{\varepsilon, \beta}^{\zeta, N}[L]$. Therefore by (20) and Axiom 3(i), we deduce that for all $L \in[N]$,

$$
o\left(\mathcal{A}_{\varepsilon, \beta}^{\zeta, N}[L]\right) \geq \omega^{\delta} .
$$

By (21) and Corollary 3.3 of $[\mathrm{G}]$ the conclusion of the proposition follows.

The hierarchy of normalized averages of a basic sequence $\left(x_{n}\right)_{n}$ constructed by I. Gasparis, E. Odell and B. Wahl satisfies the above three axioms and so it is an example of a $c_{0}$-hierarchy. This follows from Lemma 4.3 and Proposition 5.9 in [GOW] (yielding Axiom 2 and Axiom 3 respectively). In what follows, this specific $c_{0}$-hierarchy will be called the standard $c_{0^{-}}$ hierarchy generated by $\vec{x}$.

Finally, let us mention some stability properties of $c_{0}$-hierarchies. Fix a normalized basic sequence $\vec{x}=\left(x_{n}\right)_{n}$ and a $c_{0}$-hierarchy $\mathcal{H}=\left\{\left(\zeta_{n}^{L}\right)_{n}\right.$ : $\left.\zeta<\omega_{1}, L \in[\mathbb{N}]\right\}$ generated by $\vec{x}$. Let $\left(p_{n}\right)_{n}$ be a strictly increasing sequence of positive integers. Set $\vec{y}=\left(y_{n}\right)_{n}$ where $y_{n}=x_{p_{n}}$ for all $n$, and identify the linear span of $\left(x_{p_{n}}\right)_{n}$ with the linear span of $\left(y_{n}\right)_{n}$. Then for all $\zeta<\omega_{1}$, $n \in \mathbb{N}$ and $L \in[\mathbb{N}]$, we define

$$
\zeta_{n}^{L, \vec{y}}=\zeta_{n}^{p(L)}
$$

where $p(L)=\left\{p_{n}: n \in L\right\}$ for every $L \in[\mathbb{N}]$. The family

$$
\mathcal{H}^{\vec{y}}=\left\{\left(\zeta_{n}^{L, \vec{y}}\right)_{n}: \zeta<\omega_{1}, L \in[\mathbb{N}]\right\}
$$

will be called the $\vec{y}$-subhierarchy of $\mathcal{H}$. It is easy to verify that $\mathcal{H}^{\vec{y}}$ satisfies Axioms $1-3$ and therefore $\mathcal{H}^{\vec{y}}$ is a $c_{0}$-hierarchy generated by the basic sequence $\vec{y}$.

For a given ordinal $\xi<\omega_{1}$, we may also define the $\xi$-shift of $\mathcal{H}$ to be the set

$$
\mathcal{H}^{\xi}=\left\{\left(\zeta_{n}^{\xi, L}\right)_{n}: \zeta<\omega_{1}, L \in[\mathbb{N}]\right\}
$$

where $\zeta_{n}^{\xi, L}=[\xi+\zeta]_{n}^{L}$ for all $\zeta<\omega_{1}, n \in \mathbb{N}$ and $L \in[\mathbb{N}]$. Again it is easily checked that $\mathcal{H}^{\xi}$ is a $c_{0}$-hierarchy generated by $\vec{x}$.

5. Properties of $c_{0}$-hierarchies in $C(\mathcal{K})$ spaces. Fix a compact Hausdorff space $\mathcal{K}$, a normalized basic sequence $\left(f_{n}\right)_{n}$ in $C(\mathcal{K})$ and a $c_{0^{-}}$ hierarchy $\mathcal{H}=\left\{\left(\zeta_{n}^{L}\right)_{n}: \zeta<\omega_{1}, L \in[\mathbb{N}]\right\}$ generated by $\left(f_{n}\right)_{n}$.

Definition 19. Let $\mathcal{L}$ be a closed subset of $\mathcal{K}, N \in[\mathbb{N}]$ and $\zeta<\omega_{1}$. Then $\mathcal{L}$ will be called $(\zeta,[N])$-norming if there is a constant $c>0$ such that $\left\|\left.u\right|_{\mathcal{L}}\right\| \geq c$ for every $\zeta$-block $u$ with $\operatorname{supp} u \subseteq N$.

Notice that if $\mathcal{L}$ is $(\zeta,[N])$-norming then it is $(\zeta,[M])$-norming for all $M \in[N]$.

In the next series of lemmas, $\mathcal{L}$ will denote a closed subset of $\mathcal{K}$. 
Lemma 20. Let $\zeta<\omega_{1}$ and $N \in[\mathbb{N}]$. Suppose that there is no $N^{\prime} \in[N]$ such that $\mathcal{L}$ is $\left(\zeta,\left[N^{\prime}\right]\right)$-norming. Then given $\varepsilon>0$ there exists $M \in[N]$ such that $\sum_{n}\left\|\left.u_{n}\right|_{\mathcal{L}}\right\|<\varepsilon$ for every $(\zeta,[M])$-sequence $\left(u_{n}\right)_{n}$.

Proof. Let $\varepsilon>0$ and choose a summable sequence $\left(\varepsilon_{n}\right)_{n}$ of positive scalars such that $\sum_{n} \varepsilon_{n}<\varepsilon$. For each $n \in \mathbb{N}$, set $\mathcal{X}_{n}=\left\{L \in[\mathbb{N}]:\left\|\left.\zeta_{1}^{L}\right|_{\mathcal{L}}\right\|\right.$ $\left.<\varepsilon_{n}\right\}$. Fix $n \in \mathbb{N}$. By Axiom 2 we know that $\mathcal{X}_{n}$ is a clopen subset of $[\mathbb{N}]$ and therefore it has the Ramsey property. Also by our assumption there is no $N^{\prime} \in[N]$ such that $\left[N^{\prime}\right] \cap \mathcal{X}_{n}=\emptyset$ and so for every $N^{\prime} \in[N]$ there is $N^{\prime \prime} \in\left[N^{\prime}\right]$ such that $\left[N^{\prime \prime}\right] \subseteq \mathcal{X}_{n}$. Using this we construct by induction a decreasing sequence $N \supseteq N_{1} \supseteq N_{2} \supseteq \cdots$ of infinite subsets of $N$ such that $\left[N_{n}\right] \subseteq \mathcal{X}_{n}$ for every $n \in \mathbb{N}$. It is now easy to see that every diagonalization $M$ of $\left(N_{n}\right)_{n}$ satisfies the conclusion of the lemma.

Lemma 21. Let $\beta<\gamma$ be countable ordinals and $N \in[\mathbb{N}]$. Suppose that $\mathcal{L}$ is $(\gamma,[N])$-norming. Then there exists $M \in[N]$ such that $\mathcal{L}$ is $(\beta,[M])$ norming.

Proof. Assume on the contrary that there is no such $M \in[N]$. Let $c>0$ be such that $\left\|\left.u\right|_{\mathcal{L}}\right\| \geq c$ for every $\gamma$-block $u$ supported by $N$ and choose $\varepsilon>0$. By Lemma 20 and Axiom 3, there is an $N_{1} \in[N]$ satisfying the following:

(i) For every $\left(\beta,\left[N_{1}\right]\right)$-sequence $\left(b_{n}\right)_{n}, \sum_{n}\left\|\left.b_{n}\right|_{\mathcal{L}}\right\|<\varepsilon$.

(ii) Every $\gamma$-block supported by $N_{1}$ admits an $(\varepsilon, \beta)$-analysis.

Pick a $\gamma$-block $u$ supported by $N_{1}$. Then by (ii) there exist $\beta$-blocks $b_{1}<$ $\cdots<b_{n}$ supported by $N_{1}$ and scalars $\lambda_{1}, \ldots, \lambda_{n}$ such that

$$
\left\|u-\sum_{i=1}^{n} \lambda_{i} b_{i}\right\|<\varepsilon
$$

Let $C_{1}, C_{2}$ be the constants of Axiom 1. We may assume that $\left(f_{n}\right)_{n \in N_{1}}$ (and therefore $\left.\left(b_{i}\right)_{i=1}^{n}\right)$ is 2-basic. If $\varepsilon<C_{2}$ then $\left\|\sum_{i=1}^{n} \lambda_{i} b_{i}\right\|<2 C_{2}$ and so

$$
\max \left\{\left|\lambda_{i}\right|: 1 \leq i \leq n\right\}<8 C_{2} / C_{1} .
$$

Since

$$
\left\|\left.u\right|_{\mathcal{L}}-\left.\sum_{i=1}^{n} \lambda_{i} b_{i}\right|_{\mathcal{L}}\right\| \leq\left\|u-\sum_{i=1}^{n} \lambda_{i} b_{i}\right\|<\varepsilon
$$

we have

$$
c \leq\left\|\left.u\right|_{\mathcal{L}}\right\|<\varepsilon+\sum_{i=1}^{n}\left|\lambda_{i}\right|\left\|\left.b_{i}\right|_{\mathcal{L}}\right\|<\left(1+8 C_{2} / C_{1}\right) \varepsilon,
$$

which is a contradiction for $\varepsilon$ small enough.

Lemma 22. Suppose that $\left(f_{n}\right)_{n}$ is in addition shrinking. Let $\beta<\gamma$ be countable ordinals and $N \in[\mathbb{N}]$. Assume the following: 
(i) $\mathcal{L}$ is $(\beta,[N])$-norming.

(ii) There is no $N^{\prime} \in[N]$ such that $\mathcal{L}$ is $\left(\gamma,\left[N^{\prime}\right]\right)$-norming.

Then given $\varepsilon>0$ there exists $M \in[N]$ such that every $\gamma$-block supported by $M$ admits an $(\varepsilon, \beta)$-analysis of weight smaller than $\varepsilon$.

Proof. Since $\mathcal{L}$ is assumed to be $(\beta,[N])$-norming there is a constant $c>0$ such that $\left\|\left.b\right|_{\mathcal{L}}\right\| \geq c$ for every $\beta$-block $b$ supported by $N$. We set

$$
\mathcal{X}=\left\{L \in[\mathbb{N}]:\left(\left.\beta_{n}^{L}\right|_{\mathcal{L}}\right)_{n} \text { is a 2-basic sequence }\right\} .
$$

Clearly $\mathcal{X}$ is a closed subset of $[\mathbb{N}]$ and hence it has the Ramsey property. Also since $\left(f_{n}\right)_{n}$ is shrinking, $\left(\left.\beta_{n}^{L}\right|_{\mathcal{L}}\right)_{n}$ is a seminormalized weakly null sequence for every $L \in[\mathbb{N}]$. Therefore by Axiom $2, \mathcal{X}$ is cofinal in $[N]$ and so there exists $N^{\prime} \in[N]$ such that $\left[N^{\prime}\right] \subseteq \mathcal{X}$.

By Lemma 20 and Axiom 3, we can choose $M \in\left[N^{\prime}\right]$ such that for every $\gamma$-block $u$ supported by $M$,

$$
\left\|\left.u\right|_{\mathcal{L}}\right\|<\varepsilon c / 8 \text { and } u \text { admits an }(\varepsilon c / 8, \beta) \text {-analysis. }
$$

We claim that $M$ satisfies the conclusion of the lemma. Indeed, let $u$ be a $\gamma$-block supported by $M$. Then there exist $\beta$-blocks $b_{1}<\cdots<b_{n}$ supported by $M$ and scalars $\lambda_{1}, \ldots, \lambda_{n}$ such that

$$
\left\|\left.u\right|_{\mathcal{L}}-\left.\sum_{i=1}^{n} \lambda_{i} b_{i}\right|_{\mathcal{L}}\right\| \leq\left\|u-\sum_{i=1}^{n} \lambda_{i} b_{i}\right\|<\varepsilon c / 8 .
$$

Hence

$$
\left\|\left.\sum_{i=1}^{n} \lambda_{i} b_{i}\right|_{\mathcal{L}}\right\| \leq\left\|\left.u\right|_{\mathcal{L}}\right\|+\varepsilon c / 8<\varepsilon c / 4 .
$$

Since $\left(\left.b_{i}\right|_{\mathcal{L}}\right)_{i=1}^{n}$ is 2-basic we deduce that $\max \left\{\left|\lambda_{i}\right|\right\}_{i=1}^{n}<\varepsilon$, and the proof is complete.

Definition 23. Let $\mathcal{L}$ be a closed subset of $\mathcal{K}, N \in[\mathbb{N}]$ and $\zeta<\omega_{1}$. Then $\mathcal{L}$ will be called $\left(\zeta,[N], c_{0}\right)$-norming if there is a constant $C>0$ such that for every $(\zeta,[N])$-sequence $\left(u_{n}\right)_{n},\left(\left.u_{n}\right|_{\mathcal{L}}\right)_{n}$ is $C$-equivalent to the usual basis of $c_{0}$.

Proposition 24. Let $\mathcal{L}$ be a closed subset of $\mathcal{K}, N \in[\mathbb{N}]$ and $\zeta<\omega_{1}$. Then there exists $M \in[\mathbb{N}]$ such that one of the following mutually exclusive conditions holds:

(i) $\mathcal{L}$ is $\left(\zeta,[M], c_{0}\right)$-norming.

(ii) There is no $(\zeta,[M])$-sequence $\left(u_{n}\right)_{n}$ such that $\left(\left.u_{n}\right|_{\mathcal{L}}\right)_{n}$ is equivalent to the usual basis of $c_{0}$.

Proof. For each $k \in \mathbb{N}$, set $\mathcal{X}_{k}=\left\{L \in[\mathbb{N}]:\left(\left.\zeta_{n}^{L}\right|_{\mathcal{L}}\right)_{n}\right.$ is $k$-equivalent to the usual basis of $\left.c_{0}\right\}$. 
By Axiom 2, $\mathcal{X}_{k}$ is a closed subset of $[\mathbb{N}]$ and therefore it has the Ramsey property. If there exist $M \in[N]$ and $k \in \mathbb{N}$ such that $[M] \subseteq \mathcal{X}_{k}$ then we obtain the first alternative of the proposition. Otherwise we can construct by induction a decreasing sequence $N \supseteq M_{1} \supseteq M_{2} \supseteq \cdots$ of infinite subsets of $N$ such that $\left[M_{k}\right] \cap \mathcal{X}_{k}=\emptyset$ for every $k$. Then it is easily checked that for every diagonalization $M$ of $\left(M_{k}\right)_{k}$, the second alternative of the proposition is satisfied.

Lemma 25. Let $\beta<\gamma$ be countable ordinals and $N \in[\mathbb{N}]$. Assume that $\mathcal{L}$ is both $\left(\beta,[N], c_{0}\right)$-norming and $(\gamma,[N])$-norming. Then there exists $M \in[N]$ such that $\mathcal{L}$ is $\left(\gamma,[M], c_{0}\right)$-norming.

Proof. Since $\mathcal{L}$ is $\left(\beta,[N], c_{0}\right)$-norming there is a constant $C>0$ such that for every $(\beta,[N])$-sequence $\left(b_{n}\right)_{n}$,

$$
\left(\left.b_{n}\right|_{\mathcal{L}}\right)_{n} \text { is } C \text {-equivalent to the usual basis of } c_{0},
$$

and therefore $\left(\left.b_{n}\right|_{\mathcal{L}}\right)_{n}$ is a $C^{2}$-basic sequence. Also since $\mathcal{L}$ is $(\gamma,[N])$-norming there is a constant $c>0$ such that for every $\gamma$-block $u$ supported by $N$,

$$
\left\|\left.u\right|_{\mathcal{L}}\right\| \geq c .
$$

For every $n \in \mathbb{N}$, we set $\varepsilon_{n}=c / 2^{n+2} C^{2}$. Applying Axiom 3 we construct by induction a decreasing sequence $N \supseteq N_{1} \supseteq N_{2} \supseteq \cdots$ of infinite subsets of $N$ such that for every $n \in \mathbb{N}$ and every $\gamma$-block $u$ supported by $N_{n}, u$ admits an $\left(\varepsilon_{n}, \beta\right)$-analysis. Let $M$ be a diagonalization of $\left(N_{n}\right)_{n}$. We claim that $\mathcal{L}$ is $\left(\gamma,[M], c_{0}\right)$-norming.

Indeed, let $\left(u_{n}\right)_{n}$ be a $(\gamma,[M])$-sequence. By the construction of $M$ it is easy to see that there exist a $(\beta,[M])$-sequence $\left(b_{n}\right)_{n}$, a sequence $\left(\lambda_{n}\right)_{n}$ of scalars, a sequence $F_{1}<F_{2}<\cdots$ of finite subsets of $\mathbb{N}$ and a block subsequence $\left(w_{n}\right)_{n}$ of $\left(b_{n}\right)_{n}$ such that for every $n \in \mathbb{N}$,

$$
w_{n}=\sum_{i \in F_{n}} \lambda_{i} b_{i} \text { and }\left\|u_{n}-w_{n}\right\|<\varepsilon_{n} .
$$

Therefore for every $n$,

$$
\left\|\left.u_{n}\right|_{\mathcal{L}}-\left.w_{n}\right|_{\mathcal{L}}\right\|<\varepsilon_{n}
$$

and so

$$
\left.\sum_{n}|| u_{n}\right|_{\mathcal{L}}-\left.w_{n}\right|_{\mathcal{L}} \|<\frac{c}{4 C^{2}}
$$

Fix $n \in \mathbb{N}$. Then by $(23)$ and $(25)$,

$$
c / 2<\left\|\left.w_{n}\right|_{\mathcal{L}}\right\|<3 c / 2 .
$$

Set $\mu_{n}=\max \left\{\left|\lambda_{i}\right|: i \in F_{n}\right\}$. By (22) we get

$$
\frac{1}{C} \mu_{n} \leq\left\|w_{n} \mid \mathcal{L}\right\| \leq C \mu_{n}
$$


and therefore (27) gives

$$
\frac{c}{2 C}<\mu_{n}<\frac{3 c C}{2} .
$$

Let $\left(a_{n}\right)_{n} \in c_{00}(\mathbb{N})$. Then by $(24)$ and $(28)$,

$$
\frac{c}{2 C^{2}} \max _{n}\left|a_{n}\right|<\left\|\sum a_{n} w_{n}\left|\mathcal{L} \|<\frac{3 c C^{2}}{2} \max _{n}\right| a_{n} \mid\right.
$$

and so by (26) we conclude that

$$
\frac{c}{4 C^{2}} \max _{n}\left|a_{n}\right|<\left\|\sum a_{n} u_{n}\left|\mathcal{L} \|<2 c C^{2} \max _{n}\right| a_{n} \mid\right. \text {. }
$$

Therefore setting $C^{\prime}=\max \left\{4 C^{2} / c, 2 c C^{2}\right\}$ we find that every $(\gamma,[M])$ sequence is $C^{\prime}$-equivalent to the usual basis of $c_{0}$, and the proof is complete.

The next two propositions are essentially from [GOW] (Lemma 4.6 and Theorem 5.1 respectively). The first is proved similarly to Proposition 24 above. Since the proof of the second one is nontrivial, for the sake of completeness we reproduce it here.

Proposition 26. Let $\vec{x}=\left(x_{n}\right)_{n}$ be a normalized basic sequence in a Banach space $X$ and $\mathcal{H}=\left\{\left(\zeta_{n}^{L}\right)_{n}: \zeta<\omega_{1}, L \in[\mathbb{N}]\right\}$ be a c $c_{0}$-hierarchy generated by $\vec{x}$. Let $\zeta, \delta$ be countable ordinals with $\delta \geq 1$ and $N \in[\mathbb{N}]$. Then there exists $M \in[\mathbb{N}]$ such that one of the following two mutually exclusive alternatives holds:

(i) There exists a constant $C>0$ such that every $(\zeta,[M])$-sequence is a $c_{0}^{\delta}$-spreading model with constant $C$.

(ii) There is no $(\zeta,[M])$-sequence which is a $c_{0}^{\delta}$-spreading model.

Proposition 27. Let $\vec{x}=\left(x_{n}\right)_{n}$ be a normalized shrinking basic sequence in a Banach space $X$ and $\mathcal{H}=\left\{\left(\zeta_{n}^{L}\right)_{n}: \zeta<\omega_{1}, L \in[\mathbb{N}]\right\}$ be a $c_{0}$-hierarchy generated by $\vec{x}$. Let $\gamma=\beta+\delta$ be countable ordinals with $\delta \geq 1$ and $N \in[\mathbb{N}]$ be such that there is no $(\beta,[N])$-sequence which is a $c_{0}^{\delta}$-spreading model. Then given $\varepsilon>0$ there exists $M \in[N]$ such that every $\gamma$-block supported by $M$ admits an $(\varepsilon, \beta)$-analysis of weight smaller than $\varepsilon$.

Proof. Let $\varepsilon>0$ and define

$\mathcal{X}=\left\{L \in[\mathbb{N}]: \gamma_{1}^{L}\right.$ admits an $(\varepsilon, \beta)$-analysis of weight smaller than $\left.\varepsilon\right\}$.

By Axiom 2 the set $\mathcal{X}$ is a closed subset of $[\mathbb{N}]$ and hence it has the Ramsey property. Therefore there exists $M \in[N]$ such that either $[M] \subseteq \mathcal{X}$ or $[M] \cap \mathcal{X}=\emptyset$. Since by Axiom 2 every $\gamma$-block supported by $M$ is of the form $\gamma_{1}^{L}$ for some $L \in[M]$, if $[M] \subseteq \mathcal{X}$ the conclusion of the proposition follows immediately. So we must show that the other case is impossible. Therefore, suppose that $[M] \cap \mathcal{X}=\emptyset$. 
For every $L \in[\mathbb{N}]$, we will denote by $n_{L}$ the unique integer such that $\left\{\min \operatorname{supp} \beta_{i}^{L}: i=1, \ldots, n_{L}\right\}$ is a maximal member of $\mathcal{S}_{\delta}$. We may assume that $\left(x_{n}\right)_{n \in M}$ is 2 -basic and that $\varepsilon<\min \left\{1, C_{2}\right\}$ (where $C_{1}, C_{2}$ are the constants defined in Axiom 1). We define $\mathcal{Y} \subseteq[\mathbb{N}]$ to be the set of all $L \in[\mathbb{N}]$ for which there exist scalars $\mu_{1}^{L}, \ldots, \mu_{n_{L}}^{L}$ such that:

(1) for every $i=1, \ldots, n_{L},\left|\mu_{i}^{L}\right| \in\left[\varepsilon / 2,8 C_{2} / C_{1}\right]$,

(2) $\left\|\sum_{i=1}^{n_{L}} \mu_{i}^{L} \beta_{i}^{L}\right\| \leq 5 C_{2}$.

Claim. The set $\mathcal{Y}$ is cofinal in $[M]$.

Proof. Let $M^{\prime} \in[M]$. By Axiom 3 and Proposition 18, we may assume the following:

(i) Every $\gamma$-block supported by $M^{\prime}$ admits an $(\varepsilon / 2, \beta)$-analysis.

(ii) There exists $L^{\prime} \in\left[M^{\prime}\right]$ such that for every $G \in \mathcal{S}_{\delta}\left[L^{\prime}\right]$ the set $G \backslash\{\min G\}$ is an $(\varepsilon / 2, \beta)$-admissible set resulting from a $\gamma$-block supported by $M^{\prime}$.

Set $m_{1}=\min \operatorname{supp} \beta_{1}^{L^{\prime}}$ and choose a maximal member $G$ of $\mathcal{S}_{\delta}\left[L^{\prime}\right]$ such that $m_{1}=\min G$ and $\operatorname{supp} \beta_{1}^{L^{\prime}}<G \backslash\left\{m_{1}\right\}$. Notice that the stability properties of $\mathcal{S}_{\delta}$ imply that $G$ is a maximal member of $\mathcal{S}_{\delta}$. By the property of $L^{\prime}$ there is a $\gamma$-block $u$ supported by $M^{\prime}, \beta$-blocks $b_{1}<\cdots<b_{n}$ of $\vec{x}$ and scalars $\lambda_{1}, \ldots, \lambda_{n}$ such that
(a) $\bigcup_{i=1}^{n} \operatorname{supp} b_{i} \subseteq \operatorname{supp} u$.
(b) $G \backslash\left\{m_{1}\right\} \subseteq\left\{\min \operatorname{supp} b_{i}: 1 \leq i \leq n\right\}$.
(c) $\left\|u-\sum_{i=1}^{n} \lambda_{i} b_{i}\right\|<\varepsilon / 2$.
(d) ||$\lambda_{i}|-| \lambda_{j}||<\varepsilon / 2$ for all $1 \leq i, j \leq n$.

Since $M^{\prime} \notin \mathcal{X}$, it follows that $\max \left\{\left|\lambda_{i}\right|: 1 \leq i \leq n\right\} \geq \varepsilon$ and so by condition (d) we obtain

$$
\left|\lambda_{i}\right|>\varepsilon / 2 \quad \text { for all } i=1, \ldots, n \text {. }
$$

We also observe that there exists a $k \leq n$ such that $\left\{m_{1}\right\} \cup\left\{\min \operatorname{supp} b_{i}\right.$ : $1 \leq i \leq k\}$ is a maximal member of $\mathcal{S}_{\delta}$. Indeed, if this does not happen then the stability properties of $\mathcal{S}_{\delta}$ would imply that $\left\{m_{1}\right\} \cup\left\{\min \operatorname{supp} b_{i}: 1 \leq\right.$ $i \leq n\}$ is a proper initial segment of a maximal member $G^{\prime}$ of $\mathcal{S}_{\delta}$. But then $G \subseteq\left\{m_{1}\right\} \cup\left\{\min \operatorname{supp} b_{i}: 1 \leq i \leq n\right\} \subsetneq G^{\prime}$, which is impossible since both $G$ and $G^{\prime}$ are maximal members of $\mathcal{S}_{\delta}$.

By (c) we get

$$
\left\|\sum_{i=1}^{n} \lambda_{i} b_{i}\right\|<2 C_{2},
$$

and so since $\left(b_{i}\right)_{i=1}^{n}$ is a block subsequence of the 2-basic sequence $\vec{x}$ we conclude that

$$
\left|\lambda_{i}\right| \leq 8 C_{2} / C_{1} \quad \text { for all } i=1, \ldots, n
$$


and

$$
\left\|\sum_{i=1}^{k} \lambda_{i} b_{i}\right\|<4 C_{2}
$$

Choose an $L \in\left[M^{\prime}\right] \operatorname{such}$ that $\operatorname{supp} \beta_{1}^{L^{\prime}} \cup \bigcup_{i=1}^{k} \operatorname{supp} b_{i}$ is an initial segment of $L$. We set $m_{i+1}=\min \operatorname{supp} b_{i}$ for all $i=1, \ldots, k$. Then by (b) and Axiom 2, we obtain $\beta_{1}^{L}=\beta_{1}^{L^{\prime}}$ and $\beta_{i+1}^{L}=b_{i}$ for all $i=1, \ldots, k$. Furthermore $\left\{m_{1}, \ldots, m_{k+1}\right\}$ is a maximal member of $\mathcal{S}_{\delta}$ and so $k+1=n_{L}$. Setting $\mu_{1}^{L}=\varepsilon / 2$ and $\mu_{i+1}^{L}=\lambda_{i}$ for all $i=1, \ldots, k$, it is easy to check using (29), (30) and (31) above that conditions (1) and (2) of the definition of $\mathcal{Y}$ are fulfilled and therefore $L \in \mathcal{Y}$. Hence for every $M^{\prime} \in[M]$ there exists $L \in\left[M^{\prime}\right] \cap \mathcal{Y}$, and the proof of the claim is complete.

Clearly $\mathcal{Y}$ is a clopen subset of $[\mathbb{N}]$ and therefore it has the Ramsey property. By the above claim there exists $L \in[M]$ such that $[L] \subseteq \mathcal{Y}$. Since $\left(x_{n}\right)_{n}$ is shrinking, the seminormalized sequence $\left(\beta_{n}^{L}\right)_{n}$ is weakly null. By Elton's theorem [E] (see also [AG], [AGR], [O1]), we may assume that $\left(\beta_{n}^{L}\right)_{n}$ is a nearly unconditional sequence. Hence there is a constant $C>0$ such that for every $\left(a_{n}\right)_{n} \in c_{00}(\mathbb{N})$ with $\max _{n}\left|a_{n}\right| \leq 8 C_{2} / C_{1}$ and every $G \subseteq\left\{n \in \mathbb{N}:\left|a_{n}\right| \geq \varepsilon / 2\right\}$,

$$
\left\|\sum_{n \in G} a_{n} \beta_{n}^{L}\right\| \leq C\left\|\sum_{n} a_{n} \beta_{n}^{L}\right\| .
$$

We now claim that the block sequence $\left(\beta_{n}^{L}\right)_{n}$ is a $c_{0}^{\delta}$-spreading model, which of course yields a contradiction.

Indeed, let $F \subseteq \mathbb{N}$ be such that $\left\{\min \operatorname{supp} \beta_{n}^{L}: n \in F\right\}$ is a maximal member of $\mathcal{S}_{\delta}$. Let $L_{1} \in[L]$ be such that $\bigcup_{n \in F} \operatorname{supp} \beta_{n}^{L}$ is an initial segment of $L_{1}$. Set $F=\left\{n_{1}, \ldots, n_{k}\right\}$. Then by Axiom 2 , we have $\beta_{i}^{L_{1}}=\beta_{n_{i}}^{L}$ for all $i=$ $1, \ldots, k$ and so $k=n_{L_{1}}$. Since $L_{1} \in[L] \subseteq \mathcal{Y}$, there are scalars $\mu_{1}, \ldots, \mu_{k}$ such that $\left|\mu_{i}\right| \in\left[\varepsilon / 2,8 C_{2} / C_{1}\right]$ for every $i=1, \ldots, k$ and $\left\|\sum_{i=1}^{k} \mu_{i} \beta_{i}^{L_{1}}\right\| \leq 5 C_{2}$.

Let $\left(a_{n}\right)_{n} \in c_{00}(\mathbb{N})$ be defined by $a_{n_{i}}=\mu_{i}$ for all $i=1, \ldots, k$ and $a_{n}=0$ for $n \notin F$. Then by the above,

$$
\left\|\sum_{n} a_{n} \beta_{n}^{L}\right\|=\left\|\sum_{i=1}^{k} a_{n_{i}} \beta_{n_{i}}^{L}\right\|=\left\|\sum_{i=1}^{k} \mu_{i} \beta_{i}^{L_{1}}\right\| \leq 5 C_{2},
$$

and so by (32), for every sequence $\left(\sigma_{n}\right)_{n}$ of signs,

$$
\left\|\sum_{n \in F} \sigma_{n} a_{n} \beta_{n}^{L}\right\| \leq 10 C C_{2}
$$

It follows that for every $x^{*} \in X^{*}$ with $\left\|x^{*}\right\| \leq 1$, we have $\sum_{n \in F}\left|x^{*}\left(\beta_{n}^{L}\right)\right| \leq C^{\prime}$ where $C^{\prime}=20 C C_{2} / \varepsilon$, and so $\left(\beta_{n}^{L}\right)_{n}$ is a $c_{0}^{\delta}$-spreading model with constant $C^{\prime}$. 
Therefore the only possible case is that $[M] \subseteq \mathcal{X}$, and the proof of the proposition is complete.

Lemma 28. Suppose that $\left(f_{n}\right)_{n}$ is in addition shrinking. Let $\gamma=\beta+\delta$ be countable ordinals with $\delta \geq 1$ and $N \in[\mathbb{N}]$. Assume that $\mathcal{L}$ is both $\left(\beta,[N], c_{0}\right)$-norming and $(\gamma,[N])$-norming. Then there exists $M \in[N]$ such that every $(\beta,[M])$-sequence is a $c_{0}^{\delta}$-spreading model.

Proof. Assume on the contrary that there is no such $M$. Then by Proposition 26 there exists $N_{1} \in[N]$ such that there is no $\left(\beta,\left[N_{1}\right]\right)$-sequence which is a $c_{0}^{\delta}$-spreading model. By our assumptions there are constants $c, C>0$ such that for every $\gamma$-block $u$ supported by $N_{1},\left\|\left.u\right|_{\mathcal{L}}\right\| \geq c$, and for every $\left(\beta,\left[N_{1}\right]\right)$-sequence $\left.\left(b_{n}\right)_{n},\left(b_{n} \mid \mathcal{L}\right)_{n}\right)_{n}$ is $C$-equivalent to the usual basis of $c_{0}$.

Let $\varepsilon>0$. By Proposition 27 we may suppose that every $\gamma$-block supported by $N_{1}$ admits an $(\varepsilon, \beta)$-analysis of weight smaller than $\varepsilon$. Let $u$ be a $\gamma$-block supported by $N_{1}$. Then there exist $\beta$-blocks $b_{1}<\cdots<b_{n}$ supported by $N_{1}$ and scalars $\lambda_{1}, \ldots, \lambda_{n}$ such that $\left\|u-\sum_{i=1}^{n} \lambda_{i} b_{i}\right\|<\varepsilon$ and $\max \left\{\left|\lambda_{i}\right|: 1 \leq i \leq n\right\}<\varepsilon$. Therefore

$$
c \leq\left\|\left.u\right|_{\mathcal{L}}\right\|<\left\|\left.u\right|_{\mathcal{L}}-\left.\sum_{i=1}^{n} \lambda_{i} b_{i}\right|_{\mathcal{L}}\right\|+\left\|\left.\sum_{i=1}^{n} \lambda_{i} b_{i}\right|_{\mathcal{L}}\right\|<(1+C) \varepsilon,
$$

which is a contradiction for small enough $\varepsilon>0$.

6. The main theorem. Fix a compact Hausdorff space $\mathcal{K}$, a normalized shrinking basic sequence $\vec{f}=\left(f_{n}\right)_{n}$ in $C(\mathcal{K})$ and a $c_{0}$-hierarchy $\mathcal{H}=\left\{\left(\zeta_{n}^{L}\right)_{n}\right.$ : $\left.L \in[\mathbb{N}], \zeta<\omega_{1}\right\}$ generated by $\vec{f}$.

Let $\mathcal{L}$ be a countable compact metric space and $\mathcal{F}$ be a compact and hereditary family of finite subsets of $\mathbb{N}$. We will say that $\mathcal{L}$ is representable by $\mathcal{F}$ if $\mathcal{L}$ is homeomorphic to $\mathcal{F}$.

Recall that every countable compact metric space is homeomorphic to the ordinal $\left(\omega^{\xi} \cdot n\right)+1$ for some $\xi<\omega_{1}$ and $n \in \mathbb{N}$ (see [MS]). Hence, using standard constructions of compact and hereditary families of transfinite order, it follows that for every countable compact metric space $\mathcal{L}$ there exists a compact and hereditary family $\mathcal{F}$ of finite subsets of $\mathbb{N}$ such that $\mathcal{L}$ is representable by $\mathcal{F}$.

TheOrem 29. Let $\mathcal{L}$ be a countable closed subset of $\mathcal{K}, \gamma$ be a countable ordinal and $N_{0} \in[\mathbb{N}]$. Assume that:

(i) $\mathcal{L}$ is representable by a compact and hereditary family $\mathcal{F}$ such that $\mathcal{F} \subseteq \mathcal{S}_{\gamma} * k$ for some $k \in \mathbb{N}$.

(ii) There is a constant $c>0$ such that $\left\|\left.u\right|_{\mathcal{L}}\right\| \geq c$ for every $\gamma$-block $u$ supported by $N_{0}$. 
Then there exist $\beta \leq \gamma, M \in\left[N_{0}\right]$ and $C>0$ such that for all $L \in[M]$, $\left(\left.\beta_{n}^{L}\right|_{\mathcal{L}}\right)_{n}$ is $C$-equivalent to the usual basis of $c_{0}$.

In order to estimate the ordinal $\beta$ in the above theorem, we define an index (which is actually the Schreier norming index for $(\zeta,[N])$-sequences) as follows. Identifying $\mathcal{F}$ with $\mathcal{L}$ we may assume that $\mathcal{F} \subseteq \mathcal{K}$. Under this convention and using the terminology of the previous section, for every countable ordinal $\zeta \leq \gamma$ and every $N \in\left[N_{0}\right]$ we define

$$
\begin{array}{r}
\mathbf{b}(\zeta,[N])=\min \left\{\xi \leq \gamma: \text { there exist } N^{\prime} \in[N] \text { and } l \in \mathbb{N}\right. \\
\text { such that } \left.\mathcal{F} \cap\left(\mathcal{S}_{\xi} * l\right) \text { is }\left(\zeta,\left[N^{\prime}\right]\right) \text {-norming }\right\} .
\end{array}
$$

By our assumptions $\mathcal{F}$ is $\left(\gamma, N_{0}\right)$-norming and so by Lemma 21 , for all $\zeta \leq \gamma$ and $N \in\left[N_{0}\right]$, there exists $N^{\prime} \in[N]$ such that $\mathcal{F}$ is $\left(\zeta,\left[N^{\prime}\right]\right)$-norming. Therefore $\mathbf{b}(\zeta,[N])$ is a well defined ordinal (which is of course smaller than or equal to $\gamma)$.

We will prove that the ordinal $\beta$ of Theorem 29 can be chosen to be $\mathbf{b}\left(\gamma, N_{0}\right)$. For this we need two propositions.

Proposition 30. Let $\zeta \leq \gamma, N \in\left[N_{0}\right]$ and set $\beta=\mathbf{b}(\zeta,[N])$. Then there exists $M \in[N]$ such that for every $(\zeta,[M])$-sequence $\left(u_{n}\right)_{n},\left(\left.u_{n}\right|_{\mathcal{F}}\right)_{n}$ is a seminormalized weakly null sequence in $C(\mathcal{F})$ with $\beta\left(\left(\left.u_{n}\right|_{\mathcal{F}}\right)_{n}\right)=\beta$.

Proof. By the definition of $\mathbf{b}(\zeta,[N])$, there is an infinite subset of $N$, which for simplicity we denote again by $N$, such that $\mathcal{F} \cap\left(\mathcal{S}_{\beta} * l_{0}\right)$ is $(\zeta,[N])$ norming for some $l_{0} \in \mathbb{N}$. Let $\left(u_{n}\right)_{n}$ be a $(\zeta,[N])$-sequence. Since $\left(f_{n}\right)_{n}$ is shrinking it is clear that $\left(u_{n} \mid \mathcal{F}\right)_{n}$ is a seminormalized weakly null sequence in $C(\mathcal{F})$. If $\beta=0$ then trivially $\beta\left(\left(u_{n} \mid \mathcal{F}\right)_{n}\right)=0$. If $\beta \geq 1$ then let $\left(\left(\xi_{n}, l_{n}\right)\right)_{n}$ be an enumeration of all pairs $(\xi, l)$ with $\xi<\beta$. Applying Lemma 20 we construct by induction a decreasing sequence $N \supseteq N_{1} \supseteq N_{2} \supseteq \cdots$ of infinite subsets of $N$ such that $\lim _{m}\left\|\left.\zeta_{m}^{L}\right|_{\mathcal{F} \cap\left(\mathcal{S}_{\xi_{n}} * l_{n}\right)}\right\|=0$ for every $n \in \mathbb{N}$ and $L \in$ $\left[N_{n}\right]$. If $M$ is a diagonalization of $\left(N_{n}\right)_{n}$ then it is easy to see by Axiom 2 that $M$ satisfies the conclusion of the proposition.

Proposition 31. Let $\zeta \leq \gamma, N \in\left[N_{0}\right]$ and set $\beta=\mathbf{b}(\zeta,[N])$. Suppose that every $(\zeta,[N])$-sequence is a $c_{0}^{\delta}$-spreading model, where $\beta+\delta=\gamma$. Then there exists $M \in[N]$ such that $\mathcal{F}$ is $\left(\zeta,[M], c_{0}\right)$-norming.

Proof. By the preceding proposition we may assume that $\beta\left(\left(\left.u_{n}\right|_{\mathcal{F}}\right)_{n}\right)=\beta$ for every $(\zeta,[N])$-sequence $\left(u_{n}\right)_{n}$. Moreover (since $\mathcal{F} \subseteq \mathcal{K}$ ) we notice that $\left(u_{n} \mid \mathcal{F}\right)_{n}$ is also a $c_{0}^{\delta}$-spreading model in $C(\mathcal{F})$. Corollary 13 shows that for every $(\zeta,[N])$-sequence $\left(u_{n}\right)_{n}$ there exists a subsequence of $\left(u_{n} \mid \mathcal{F}\right)_{n}$ equivalent to the usual basis of $c_{0}$. Hence for every $L \in[N]$ there exists $L^{\prime} \in[L]$ such that $\left(\left.\zeta_{n}^{L^{\prime}}\right|_{\mathcal{F}}\right)_{n}$ is equivalent to the usual basis of $c_{0}$, and so by Proposition 24 the proof is complete.

The following is an immediate consequence of the above proposition. 
Corollary 32. Let $\zeta \leq \gamma$ and $N \in\left[N_{0}\right]$. If $\mathbf{b}(\zeta,[N])=\gamma$ then there exists $M \in[N]$ such that $\mathcal{F}$ is $\left(\zeta,[M], c_{0}\right)$-norming.

We are now ready to prove Theorem 29.

Proof of Theorem 29. We identify $\mathcal{L}$ with $\mathcal{F}$ and we assume that $\mathcal{F} \subseteq \mathcal{K}$. The proof will be done by induction on the ordinal $\gamma$ and with inductive hypothesis the following restatement of the theorem:

InDUCTIVE HYPOTHESIS. Let $\gamma<\omega_{1}$. Then for every $N_{0} \in[\mathbb{N}]$ and every compact and hereditary family $\mathcal{F}$ such that (i) $\mathcal{F} \subseteq \mathcal{S}_{\gamma} * k$ for some $k \in \mathbb{N}$ and (ii) $\mathcal{F}$ is $\left(\gamma,\left[N_{0}\right]\right)$-norming, there exist $\beta \leq \gamma$ and $M \in\left[N_{0}\right]$ such that $\mathcal{F}$ is $\left(\beta,[M], c_{0}\right)$-norming.

So fix a countable ordinal $\gamma$ and suppose that our inductive hypothesis is true for all ordinals smaller than $\gamma$. We set

$$
\beta=\mathbf{b}\left(\gamma,\left[N_{0}\right]\right)
$$

and we will show that there exists $M \in\left[N_{0}\right]$ such that $\mathcal{F}$ is $\left(\beta,[M], c_{0}\right)$ norming.

Notice that if $\beta=\gamma$ then the result follows immediately from Corollary 32 (this in particular establishes the case of $\gamma=0$ ).

So suppose that $\beta<\gamma$. By the definition of $\mathbf{b}\left(\gamma,\left[N_{0}\right]\right)$ there exist $l_{0} \in \mathbb{N}$ and $N_{0}^{\prime} \in\left[N_{0}\right]$ such that

$$
\mathcal{F}^{\prime}=\mathcal{F} \cap\left(\mathcal{S}_{\beta} * l_{0}\right) \text { is }\left(\gamma,\left[N_{0}^{\prime}\right]\right) \text {-norming. }
$$

By Lemma 21, we may assume that $\mathcal{F}^{\prime}$ is $\left(\beta,\left[N_{0}^{\prime}\right]\right)$-norming. Since $\mathcal{F}^{\prime}$ is a compact and hereditary family with $\mathcal{F}^{\prime} \subseteq \mathcal{S}_{\beta} * l_{0}$ and $\beta<\gamma$, our inductive hypothesis can be applied. Therefore there exist $\beta^{\prime} \leq \beta$ and $N \in\left[N_{0}^{\prime}\right]$ such that $\mathcal{F}^{\prime}$ is $\left(\beta^{\prime},[N], c_{0}\right)$-norming. Since $N \in\left[N_{0}^{\prime}\right]$, we find that $\mathcal{F}^{\prime}$ is $(\beta,[N])$-norming and so by Lemma 25 we may assume that

$$
\mathcal{F}^{\prime} \text { is }\left(\beta,[N], c_{0}\right) \text {-norming. }
$$

Let $\delta<\omega_{1}$ be such that $\beta+\delta=\gamma$. Since $N \in\left[N_{0}^{\prime}\right]$ and $\mathcal{F}^{\prime}$ is $\left(\gamma,\left[N_{0}^{\prime}\right]\right)$ norming, by Lemma 28 we may also suppose that

$$
\text { every }(\beta,[N]) \text {-sequence is a } c_{0}^{\delta} \text {-spreading model. }
$$

We now claim that

$$
\mathbf{b}(\beta,[N])=\beta .
$$

Indeed, recall that

$$
\begin{aligned}
& \mathbf{b}(\beta,[N])=\min \left\{\xi \leq \gamma: \text { there exist } N^{\prime} \in[N] \text { and } l \in \mathbb{N}\right. \\
&\text { such that } \left.\mathcal{F} \cap\left(\mathcal{S}_{\xi} * l\right) \text { is }\left(\beta,\left[N^{\prime}\right]\right) \text {-norming }\right\} .
\end{aligned}
$$

Since $\mathcal{F}^{\prime}=\mathcal{F} \cap\left(\mathcal{S}_{\beta} * l_{0}\right)$ is $(\beta,[N])$-norming, we have $\mathbf{b}(\beta,[N]) \leq \beta$. So it remains to show the reverse inequality. 
Assume on the contrary that $\mathbf{b}(\beta,[N])<\beta$. Then there exist $\xi<\beta$, $N^{\prime} \in[N]$ and $l \in \mathbb{N}$ such that $\mathcal{F} \cap\left(\mathcal{S}_{\xi} * l\right)$ is $\left(\beta,\left[N^{\prime}\right]\right)$-norming. On the other hand, observe that $N^{\prime} \in\left[N_{0}\right]$ and since $\xi<\beta=\mathbf{b}\left(\gamma,\left[N_{0}\right]\right)$, we conclude that there is no $N^{\prime \prime} \in\left[N^{\prime}\right]$ such that $\mathcal{F} \cap\left(\mathcal{S}_{\xi} * l\right)$ is $\left(\gamma,\left[N^{\prime \prime}\right]\right)$-norming. Therefore by Lemma 22, for every $\varepsilon>0$ there exists $M^{\prime} \in\left[N^{\prime}\right]$ such that every $\gamma$-block supported by $M^{\prime}$ admits an $(\varepsilon, \beta)$-analysis of weight smaller than $\varepsilon$. Furthermore by (36) there is a constant $C>0$ such that for every $(\beta,[N])$-sequence $\left(b_{n}\right)_{n},\left(\left.b_{n}\right|_{\mathcal{F}^{\prime}}\right)_{n}$ is $C$-equivalent to the usual basis of $c_{0}$.

Using the above and arguing as we did in the second part of the proof of Lemma 28 it follows easily that for every $\varepsilon>0$ there exists $M^{\prime} \in\left[N^{\prime}\right]$ such that $\left\|\left.u\right|_{\mathcal{F}^{\prime}}\right\| \leq \varepsilon$ for every $\gamma$-block $u$ supported by $M^{\prime}$, which is impossible by $(35)$.

By (37), (38) and Proposition 31 (with $\beta$ in place of $\zeta$ ) we obtain an $M \in[N]$ such that $\mathcal{F}$ is $\left(\beta,[M], c_{0}\right)$-norming, and the proof of the theorem is complete.

7. Consequences of the main theorem. The next theorem proved in [GOW] for the standard $c_{0}$-hierarchy is the main consequence of Theorem 29 .

THEOREM 33. Let $\xi<\omega_{1},\left(f_{n}\right)_{n}$ be a weakly null normalized basic sequence in $C\left(\omega^{\omega^{\xi}}\right)$ and $\mathcal{H}=\left\{\left(\zeta_{n}^{L}\right)_{n}: L \in \mathbb{N}, \zeta<\omega_{1}\right\}$ be a co-hierarchy generated by $\left(f_{n}\right)_{n}$. Then for every $N \in[\mathbb{N}]$ there exist $\beta \leq \xi, M \in[N]$ and $C>0$ such that for every $L \in[M]$, the sequence $\left(\beta_{n}^{L}\right)_{n}$ is $C$-equivalent to the usual basis of $c_{0}$.

Proof. A standard diagonal argument gives a $P \in[N], P=\left(p_{n}\right)_{n}$, such that for all $t \in \mathcal{K}$ the series $\sum_{n}\left|f_{p_{n}}(t)\right|$ is summable. Let $g_{n}=f_{p_{n}}$ for $n \in \mathbb{N}$ and set $\vec{g}=\left(g_{n}\right)_{n}$. By the above $\vec{g}$ is a shrinking basic sequence in $C\left(\omega^{\omega^{\xi}}\right)$.

Let $\mathcal{H}^{\vec{g}}=\left\{\left(\zeta_{n}^{L, \vec{g}}\right)_{n}: L \in[\mathbb{N}], \zeta<\omega_{1}\right\}$ be the $\vec{g}$-subhierarchy of $\mathcal{H}$ (recall that for all $L \in \mathbb{N}, n \in \mathbb{N}$ and $\zeta<\omega_{1}$, we have $\zeta_{n}^{L, \vec{g}}=\zeta_{n}^{p(L)}$ where $p(L)=$ $\left\{p_{n}: n \in L\right\}$ ). Applying Theorem 29 (for $\gamma=\xi, \mathcal{K}=\mathcal{L}=\mathcal{S}_{\xi}, N_{0}=\mathbb{N}$ and for the sequence $\vec{g}$ and the $c_{0}$-hierarchy $\mathcal{H}^{\vec{g}}$ in place of $\vec{f}$ and $\mathcal{H}$ ) we find that there exist $\beta \leq \gamma, M^{\prime} \in[\mathbb{N}]$ and a constant $C>0$ such that for every $L^{\prime} \in\left[M^{\prime}\right]$, the sequence $\left(\beta_{n}^{L^{\prime}, \vec{g}}\right)_{n}$ is $C$-equivalent to the usual basis of $c_{0}$. We set $M=p\left(M^{\prime}\right)$ and it is easy to see that $M$ satisfies the conclusion of the theorem.

As an application of Theorem 33 we obtain

Corollary 34. Let $\xi<\omega_{1}$ and $X$ be a quotient of $C\left(\omega^{\omega^{\xi}}\right)$ such that $X$ contains a sequence $\left(x_{n}\right)_{n}$ which is an $l_{1}^{\xi}$-spreading model. Then there is a block subsequence of $\left(x_{n}\right)_{n}$ equivalent to the usual basis of $c_{0}$. 
Proof. Let $Q: C\left(\omega^{\omega^{\xi}}\right) \rightarrow X$ be a continuous onto linear map. By the open mapping theorem we can choose a bounded sequence $\vec{g}=\left(g_{n}\right)_{n}$ in $C\left(\omega^{\omega^{\xi}}\right)$ such that $Q\left(g_{n}\right)=x_{n}$ for all $n \in \mathbb{N}$. Since $\omega^{\omega^{\xi}}$ is countable, we may suppose that $\left(g_{n}\right)_{n}$ is pointwise convergent. Let $\vec{f}=\left(f_{n}\right)_{n}$, where for every $n \in \mathbb{N}$,

$$
f_{n}=\frac{g_{2 n}-g_{2 n+1}}{\left\|g_{2 n}-g_{2 n+1}\right\|} .
$$

Notice that $\vec{f}$ is a normalized weakly null sequence in $C\left(\omega^{\omega^{\xi}}\right)$. Hence by passing to a subsequence, we may assume that $\vec{f}$ is in addition a basic sequence.

Let $y_{n}=Q\left(f_{n}\right)$ for all $n \in \mathbb{N}$ and set $\vec{y}=\left(y_{n}\right)_{n}$. It is clear that $\vec{y}$ is a block subsequence of $\vec{x}$ and it is easy to check that $\left(y_{n}\right)_{n}$ is also an $l_{1}^{\xi}$-spreading model. Hence there is a constant $c>0$ such that for all $F \in \mathcal{S}_{\xi}$ and scalars $\left(\lambda_{i}\right)_{i \in F}$

$$
c \sum_{i \in F}\left|\lambda_{i}\right| \leq\left\|\sum_{i \in F} \lambda_{i} y_{i}\right\| .
$$

Let $\mathcal{H}=\left\{\left(\zeta_{n}^{L}\right)_{n}: L \in \mathbb{N}, \zeta<\omega_{1}\right\}$ be a $c_{0}$-hierarchy generated by $\vec{f}$ such that $\operatorname{supp} u \in \mathcal{S}_{\zeta}$ for every $\zeta$-block $u$ (for example the standard $c_{0}$-hierarchy generated by $\vec{f}$ has this property). By Theorem 33, there exist $M \in[\mathbb{N}]$, $\beta \leq \xi$ and $C>0$ such that $\left(\beta_{n}^{M}\right)_{n}$ is $C$-equivalent to the usual basis of $c_{0}$.

Define $\vec{w}=\left(w_{n}\right)_{n}$ where $w_{n}=Q\left(\beta_{n}^{M}\right)$ for all $n$. Then $\left(w_{n}\right)_{n}$ is a block subsequence of $\left(x_{n}\right)_{n}$ and for all $\left(a_{n}\right)_{n} \in c_{00}(\mathbb{N})$, we have

$$
\left\|\sum_{n} a_{n} w_{n}\right\| \leq\|Q\|\left\|\sum_{n} a_{n} \beta_{n}^{M}\right\| \leq\|Q\| C \max _{n}\left|a_{n}\right| .
$$

Therefore to complete the proof it suffices to show that $\left(w_{n}\right)_{n}$ contains a basic subsequence. By the properties of Schreier families there exists $n_{0} \in \mathbb{N}$ such that $F \in \mathcal{S}_{\xi}$ for all $F \in \mathcal{S}_{\beta}$ with $n_{0} \leq F$. Fix $n \geq n_{0}$ and set $F=$ $\operatorname{supp} \beta_{n}^{M}$. Then $n_{0} \leq F$ and so $F \in \mathcal{S}_{\xi}$. Let $\left(\lambda_{i}\right)_{i \in F}$ be scalars such that $\beta_{n}^{M}=\sum_{i \in F} \lambda_{i} f_{i}$. Then $w_{n}=Q\left(\beta_{n}^{M}\right)=\sum_{i \in F} \lambda_{i} y_{i}$ and so by (40) we have

$$
\left\|w_{n}\right\|=\left\|\sum_{i \in F} \lambda_{i} y_{i}\right\| \geq c \sum_{i \in F}\left|\lambda_{i}\right| \geq c\left\|\beta_{n}^{M}\right\| .
$$

Hence $\left(w_{n}\right)_{n \geq n_{0}}$ is seminormalized. Since it is also weakly null, it contains a basic subsequence, and the proof is complete.

REMARK 4. The above result is related to a well known open problem, namely whether every quotient of $C(\mathcal{K})$, with $\mathcal{K}$ a countable compact metric space, is $c_{0}$-saturated. In [O2] (Theorem B) it has been shown that every quotient of the Schreier space is $c_{0}$-saturated. 
Let us also notice that since Tsirelson's space contains $l_{1}^{n}$-spreading models for all $n \in \mathbb{N}$, by Corollary 34 it follows that Tsirelson's space is not a subspace of a quotient of any $C\left(\omega^{\omega^{n}}\right)$. However, it is open if it is a subspace of a quotient of $C\left(\omega^{\omega^{\omega}}\right)$.

Fix a compact Hausdorff space $\mathcal{K}$, a normalized shrinking basic sequence $\left(f_{n}\right)_{n}$ in $C(\mathcal{K})$ and a $c_{0}$-hierarchy $\mathcal{H}=\left\{\left(\zeta_{n}^{L}\right)_{n}: L \in[\mathbb{N}], \zeta<\omega_{1}\right\}$ generated by $\left(f_{n}\right)_{n}$. In the following, for a countable compact metric space $\mathcal{L}$, we denote by $|\mathcal{L}|_{\text {CB }}^{*}$ the immediate predecessor of the Cantor-Bendixson rank of $\mathcal{L}$ (i.e. the greatest ordinal $\xi<\omega_{1}$ with $\mathcal{L}^{(\xi)} \neq \emptyset$ ).

Corollary 35. Let $\mathcal{L}$ be a countable closed subset of $\mathcal{K}$ and $\gamma$ be a countable ordinal such that $|\mathcal{L}|_{\mathrm{CB}}^{*}<\omega^{\gamma+1}$. Then for every $\xi \geq \gamma$ and every $N \in[\mathbb{N}]$ there exists $M \in[N]$ such that one of the following two mutually exclusive alternatives holds:

(1) For all $L \in[M]$, the sequence $\left(\left.\xi_{n}^{L}\right|_{\mathcal{L}}\right)_{n}$ is equivalent to the usual basis of $c_{0}$.

(2) For all $L \in[M], \lim \left\|\left.\xi_{n}^{L}\right|_{\mathcal{L}}\right\|=0$.

Proof. By $[\mathrm{MS}], \mathcal{L}$ may be identified with an initial segment of $\omega^{\omega^{\gamma} \cdot k}$ for some $k \in \mathbb{N}$. Set $\mathcal{D}=\omega^{\omega^{\gamma} \cdot k} \backslash \mathcal{L}$. Replacing $\mathcal{K}$ and $\mathcal{L}$ with the direct sums $\mathcal{K} \oplus \mathcal{D}$ and $\mathcal{L} \oplus \mathcal{D}$ respectively and considering the trivial extension of each $f_{n}$ (that is, $f_{n}(\zeta)=0$ for all $\zeta \in \mathcal{D}$ ) we may assume that $\mathcal{L}=\omega^{\omega^{\gamma} \cdot k}$ for some $k \in \mathbb{N}$. Since $\mathcal{F}=\mathcal{S}_{\gamma} * k$ is homeomorphic to $\left[0, \omega^{\omega^{\gamma} \cdot k}\right]$, we see that $\mathcal{L}$ is representable by the regular family $\mathcal{S}_{\gamma} * k$.

Fix $\xi \geq \gamma$ and $N \in[\mathbb{N}]$. Then either $\mathcal{L}$ is $\left(\xi,\left[N^{\prime}\right]\right)$-norming for some $N^{\prime} \in[N]$, or there is no such $N^{\prime}$. In the first case, by Lemma 21 there is $N_{0} \in$ $\left[N^{\prime}\right]$ such that $\mathcal{L}$ is $\left(\gamma,\left[N_{0}\right]\right)$-norming. Applying successively Theorem 29 and Lemma 25 yields statement (1). In the second case Lemma 20 immediately gives statement (2).

Corollary 36. Let $\mathcal{L}$ be a countable closed subset of $\mathcal{K}$ and $\gamma$ be a countable ordinal such that $|\mathcal{L}|_{\mathrm{CB}}^{*}<\omega^{\gamma+1}$. Let $\xi$ be a countable ordinal with $\xi \geq \gamma$ and $N_{0} \in[\mathbb{N}]$ be such that $\mathcal{L}$ is $\left(\xi,\left[N_{0}\right]\right)$-norming. Then there exists a countable ordinal $\beta \leq \gamma$ such that for every $\zeta \in[\beta, \xi]$ there is an $M \in\left[N_{0}\right]$ such that for all $L \in[M]$ the following are satisfied:

(1) The sequence $\left(\left.\zeta_{n}^{L}\right|_{\mathcal{L}}\right)_{n}$ is equivalent to the usual basis of $c_{0}$.

(2) The sequence $\left(\zeta_{n}^{L}\right)_{n}$ is a $c_{0}^{\delta}$-spreading model, where $\zeta+\delta=\xi$.

Proof. Since $\gamma \leq \xi$, by Lemma 21 we may suppose that $\mathcal{L}$ is $\left(\gamma,\left[N_{0}\right]\right)$ norming. As in Corollary 35, we may suppose that $\mathcal{L}$ is representable by the regular family $\mathcal{S}_{\gamma} * k$. Therefore by Theorem 29 there exist $\beta \leq \gamma$ and $N \in\left[N_{0}\right]$ such that $\mathcal{L}$ is $\left(\beta,[N], c_{0}\right)$-norming. 
Let $\zeta \in[\beta, \xi]$. Clearly $\mathcal{L}$ is $(\gamma,[N])$-norming and so by Lemma 21 , we may assume that $\mathcal{L}$ is $(\zeta,[N])$-norming. Applying successively Lemma 25 and Lemma 28 yields the result.

Corollary 37. Let $\Lambda$ be an uncountable subset of $\omega_{1}$ satisfying the following:

(i) For every $\xi \in \Lambda$ there exist a countable closed subset $\mathcal{L}_{\xi}$ of $\mathcal{K}$ and $N_{\xi} \in[\mathbb{N}]$ such that $\mathcal{L}_{\xi}$ is $\left(\xi,\left[N_{\xi}\right]\right)$-norming.

(ii) $\sup \left\{\left|\mathcal{L}_{\xi}\right|_{\text {CB }}^{*}: \xi \in \Lambda\right\}<\omega_{1}$.

Then there exists a countable ordinal $\gamma_{0}$ such that for each $\gamma \geq \gamma_{0}$ there exists $L \in[\mathbb{N}]$ such that the sequence $\left(\gamma_{n}^{L}\right)_{n}$ is equivalent to the usual basis of $c_{0}$.

Proof. Let $\gamma_{0}$ be the least countable ordinal such that $\sup \left\{\left|\mathcal{L}_{\xi}\right|_{\mathrm{CB}}^{*}\right.$ : $\xi \in \Lambda\}<\omega^{\gamma_{0}+1}$. Fix $\gamma \geq \gamma_{0}$ and for each $k \in \mathbb{N}$ define the tree

$$
\mathcal{T}_{\gamma}^{k}=\left\{\left(\gamma_{i}^{L}\right)_{i=1}^{n}: L \in[\mathbb{N}], n \in \mathbb{N} \text { and }\left(\gamma_{i}^{L}\right)_{i=1}^{n} \text { is } k \text {-equivalent to }\left(e_{i}\right)_{i=1}^{n}\right\}
$$

where $\left(e_{i}\right)_{i}$ denotes the usual basis of $c_{0}$. We have to show that there is a $k \in \mathbb{N}$ such that $\mathcal{T}_{\gamma}^{k}$ is not well founded.

Assume on the contrary that $\mathcal{T}_{\gamma}^{k}$ is well founded for every $k$. By Axiom 2 of the definition of $c_{0}$-hierarchies the family $\left\{\gamma_{i}^{L}: L \in[\mathbb{N}], i \in \mathbb{N}\right\}$ is countable. Hence for every $k, \mathcal{T}_{\gamma}^{k}$ is a countable tree and therefore its order, $o\left(\mathcal{T}_{\gamma}^{k}\right)$, must be a countable ordinal.

Set $\Lambda_{\gamma}=\{\xi: \xi \in \Lambda, \xi \geq \gamma\}$. Let $\xi \in \Lambda_{\gamma}$ and set $\xi=\gamma+\delta_{\xi}$. Since $\left|\mathcal{L}_{\xi}\right|_{\mathrm{CB}}^{*}<\omega^{\gamma+1}$ and $\mathcal{L}_{\xi}$ is $\left(\xi,\left[N_{\xi}\right]\right)$-norming, Corollary 36 shows that there exists $L \in\left[N_{\xi}\right]$ such that the sequence $\left(\gamma_{n}^{L}\right)_{n}$ is a $c_{0}^{\delta_{\xi}}$-spreading model. This implies that there exists a $k \in \mathbb{N}$ and a strictly monotone map from the tree $\left(\mathcal{S}_{\xi}, \sqsubseteq\right)$ to $\mathcal{T}_{\gamma}^{k}$, and so $o\left(\mathcal{T}_{\gamma}^{k}\right) \geq \omega^{\delta_{\xi}}$ (see $[\mathrm{K}]$ ). Since $\Lambda_{\gamma}$ is uncountable, there exists a $k \in \mathbb{N}$ such that $o\left(\mathcal{T}_{\gamma}^{k}\right) \geq \omega_{1}$, which is a contradiction.

8. Appendix. This section is devoted to the proof of Proposition 5, namely that for all countable ordinals $\xi=\beta+\delta$ and all $k \in \mathbb{N}$, the following equality holds:

$$
o\left(\left(\mathcal{S}_{\xi} * k\right) / \mathcal{S}_{\beta}\right)=\omega^{\delta} \cdot k .
$$

Before starting the proof let us show that in certain cases the order of a quotient family of the form $\mathcal{F} / \mathcal{S}_{\beta}$ may be equal to the order of the family $\mathcal{F}$.

We will need the following definition from $[\mathrm{AMT}]$. Consider an increasing map $f: \mathbb{N} \rightarrow \mathbb{N}$ and set $\mathcal{S}_{1}^{f}=\{t \subseteq \mathbb{N}:|t| \leq f(\min t)\}$. It is clear that for constant maps $f$ we get the families $[\mathbb{N}]^{\leq k}$, whereas if $f$ is the identity then we obtain the first Schreier family $\mathcal{S}_{1}$. It is easy to see that for an 
increasing map $f$ with infinite range, $\mathcal{S}_{1}^{f}$ is a regular family of order $\omega$ and so homeomorphic to $\mathcal{S}_{1}$.

Now it is not difficult to show that choosing $f$ appropriately, for example $f(n)=n^{2}$, we obtain $o\left(\mathcal{S}_{1}^{f} / \mathcal{S}_{1}\right)=\omega$. Furthermore one can define transfinite analogs of $\mathcal{S}_{1}^{f},\left\{\mathcal{S}_{\xi}^{f}\right\}_{\xi<\omega_{1}}$, in the way the Schreier families are constructed, and show by induction that $o\left(\mathcal{S}_{\beta}^{f} / \mathcal{S}_{\beta}\right)=o\left(\mathcal{S}_{\beta}^{f}\right)=\omega^{\beta}$ for every $\beta<\omega_{1}$.

We now pass to the proof of Proposition 5. We start with some preparatory lemmas. We will denote by $\mathcal{G}$ a regular family of finite subsets of $\mathbb{N}$ that covers $\mathbb{N}$.

Lemma 38. Let $M \in[\mathbb{N}]$ and $\emptyset \neq s \sqsubseteq M$. Set $L=M \backslash s, q_{\mathcal{G}}(M)=$ $\left(m_{n}\right)_{n}$ and $q_{\mathcal{G}}(L)=\left(l_{n}\right)_{n}$. Let $d$ be such that $m_{d}<\min L \leq m_{d+1}$. Then $m_{d+n-1}<l_{n} \leq m_{d+n}$ for all $n$.

Proof. Since $\min L=l_{1}$ our assumption gives $m_{d}<l_{1} \leq m_{d+1}$. If $l_{1}=$ $m_{d+1}$, then it is easy to see from the definitions that $l_{n}=m_{d+n}$ for all $n \in \mathbb{N}$. So assume that $m_{d}<l_{1}<m_{d+1}$. We show that $m_{d+1}<l_{2}<m_{d+2}$. Note first that $\left[m_{i}, m_{i+1}\right) \cap M$ and $\left[l_{i}, l_{i+1}\right) \cap M$ are maximal members of $\mathcal{G}$, for all $i \in \mathbb{N}$. If $l_{2} \leq m_{d+1}$, then $\left[l_{1}, l_{2}\right) \cap M$ is a proper subset of $\left[m_{d}, m_{d+1}\right) \cap M$. When $m_{d+2} \leq l_{2},\left[m_{d+1}, m_{d+2}\right) \cap M$ is a proper subset of $\left[l_{1}, l_{2}\right) \cap M$. In either case, maximality is violated. Repeat the same argument for $L \backslash\left[l_{1}, l_{2}\right)$ to show that $m_{d+2}<l_{3}<m_{d+3}$ and continue inductively.

Lemma 39. Let $k \in \mathbb{N}$ and $s_{1}<\cdots<s_{k}$ be finite subsets of $\mathbb{N}$. Then $q_{\mathcal{G}}\left(\bigcup_{i=1}^{k} s_{i}\right)=\bigcup_{i=1}^{k} s_{i}^{\prime}$ where $s_{1}^{\prime}<\cdots<s_{k}^{\prime}, s_{1}^{\prime}=q_{\mathcal{G}}\left(s_{1}\right)$ and for every $1<i \leq k$ either

(1) $q_{\mathcal{G}}\left(s_{i}\right) \preceq s_{i}^{\prime}$ or

(2) $q_{\mathcal{G}}\left(s_{i}\right) \backslash\left\{\max q_{\mathcal{G}}\left(s_{i}\right)\right\} \preceq s_{i}^{\prime}$.

Proof. Let $k=2$. We assume that both $s_{1}, s_{2}$ are nonempty; otherwise the result follows trivially. We choose $M \in[\mathbb{N}]$ such that $s_{1} \cup s_{2} \sqsubseteq M$ and let $q_{\mathcal{G}}(M)=\left(m_{n}\right)_{n}$. Then

$$
\begin{aligned}
q_{\mathcal{G}}\left(s_{1} \cup s_{2}\right) & =\left(s_{1} \cup s_{2}\right) \cap q_{\mathcal{G}}(M)=\left(s_{1} \cap q_{\mathcal{G}}(M)\right) \cup\left(s_{2} \cap q_{\mathcal{G}}(M)\right) \\
& =q_{\mathcal{G}}\left(s_{1}\right) \cup\left(s_{2} \cap q_{\mathcal{G}}(M)\right) .
\end{aligned}
$$

Let $d \in \mathbb{N}$ be such that $q_{\mathcal{G}}\left(s_{1}\right)=\left(m_{n}\right)_{n=1}^{d}$. Let $L=M \backslash s_{1}$ and $q_{\mathcal{G}}(L)=$ $\left(l_{n}\right)_{n}$. Then $s_{2} \sqsubseteq L, q_{\mathcal{G}}\left(s_{2}\right)=s_{2} \cap q_{\mathcal{G}}(L)$ and $m_{d}<l_{1} \leq m_{d+1}$. We set $s_{2}^{\prime}=s_{2} \cap q_{\mathcal{G}}(M)$ and we distinguish the following cases.

CASE 1: $s_{2}^{\prime}=\emptyset$. Then $q_{\mathcal{G}}\left(s_{2}\right)=\left\{l_{1}\right\}$. Indeed, otherwise $l_{2} \in q_{\mathcal{G}}\left(s_{2}\right)$. But then by Lemma 38 we have $l_{1} \leq m_{d+1}<l_{2}$ and so $m_{d+1} \in s_{2}^{\prime}$, which is a contradiction.

CASE 2: $s_{2}^{\prime} \neq \emptyset$. Let $k \in \mathbb{N}$ be such that $s_{2}^{\prime}=\left\{m_{d+1}, \ldots, m_{d+k}\right\}$. Then again by Lemma 38 we obtain 


$$
\left\{l_{1}, \ldots, l_{k}\right\} \sqsubseteq q_{\mathcal{G}}\left(s_{2}\right) \sqsubseteq\left\{l_{1}, \ldots, l_{k+1}\right\}, \quad\left\{l_{1}, \ldots, l_{k}\right\} \preceq\left\{m_{d+1}, \ldots, m_{d+k}\right\} .
$$

The above yields the assertion for $k=2$ and an easy induction completes the proof for every $k$.

Lemma 40. Let $\mathcal{F}$ be a regular family in $\mathbb{N}$.

(i) For every $k \in \mathbb{N},(\mathcal{F} * k) / \mathcal{G} \subseteq(\mathcal{F} / \mathcal{G}) * k$.

(ii) $\left(\mathcal{F} * \mathcal{S}_{1}\right) / \mathcal{G} \subseteq(\mathcal{F} / \mathcal{G}) * \mathcal{S}_{1}$.

Proof. (i) Since $(\mathcal{F} / \mathcal{G}) * k$ is spreading it is enough to show that $q_{\mathcal{G}}(s) \in$ $(\mathcal{F} / \mathcal{G}) * k$ for every $s \in \mathcal{F} * k$. So let $s \in \mathcal{F} * k$. Then there exist $d \leq k$ and members $s_{1}<\cdots<s_{d}$ of $\mathcal{F}$ such that $s=\bigcup_{i=1}^{d} s_{i}$. By Lemma 39 and since $\mathcal{F} / \mathcal{G}$ is hereditary and spreading, we conclude that $q_{\mathcal{G}}(s) \in(\mathcal{F} / \mathcal{G}) * k$, and the proof of part (i) is complete.

(ii) As above it suffices to show that $q_{\mathcal{G}}(s) \in(\mathcal{F} / \mathcal{G}) * \mathcal{S}_{1}$ for every $s \in$ $\mathcal{F} * \mathcal{S}_{1}$. Indeed, let $s \in \mathcal{F} * \mathcal{S}_{1}$. Then there is $k \in \mathbb{N}$ such that $s \in \mathcal{F} * k$ and $k \leq s$. Then by part (i), $q_{\mathcal{G}}(s) \in(\mathcal{F} / \mathcal{G}) * k$ and $k \leq q_{\mathcal{G}}(s)$ and therefore $q_{\mathcal{G}}(s) \in(\mathcal{F} / \mathcal{G}) * \mathcal{S}_{1}$.

We will also need the following general proposition. The proof of part (i) is essentially contained in [AT], whereas (ii) follows by standard arguments.

Proposition 41. (i) Let $\mathcal{F}, \mathcal{G}$ be regular families in $\mathbb{N}$. Then $o(\mathcal{G} * \mathcal{F})=$ $o(\mathcal{G}) \cdot o(\mathcal{F})$.

(ii) Let $\left(\mathcal{F}_{n}\right)_{n}$ be a sequence of regular families in $\mathbb{N}$ such that $n \leq t$ for every $n \in \mathbb{N}$ and every $t \in \mathcal{F}_{n}$. Then the family $\mathcal{F}=\bigcup_{n} \mathcal{F}_{n}$ is also regular and $o(\mathcal{F})=\sup _{n} o\left(\mathcal{F}_{n}\right)$.

Lemma 42. For all countable ordinals $\xi=\beta+\delta$ and all $k \in \mathbb{N}$,

$$
\omega^{\delta} \cdot k \leq o\left(\left(\mathcal{S}_{\xi} * k\right) / \mathcal{S}_{\beta}\right)
$$

Proof. It is clear that $\mathcal{S}_{\xi} * k \subseteq \mathcal{S}_{\beta} *\left(\left(\mathcal{S}_{\xi} * k\right) / \mathcal{S}_{\beta}\right)$ and so by the first part of the above proposition we infer that $\omega^{\xi} \cdot k \leq \omega^{\beta} \cdot o\left(\left(\mathcal{S}_{\xi} * k\right) / \mathcal{S}_{\beta}\right)$, which yields the conclusion.

LEMma 43. Let $\beta, \xi$ be countable ordinals.

(i) If $\xi<\beta$ then $o\left(\mathcal{S}_{\xi} / \mathcal{S}_{\beta}\right)<\omega$.

(ii) If $\xi \geq \beta$ then $o\left(\mathcal{S}_{\xi} / \mathcal{S}_{\beta}\right)=\omega^{\delta}$, where $\beta+\delta=\xi$.

Proof. (i) Let $\xi<\beta$. Then by the properties of the Schreier families there is an $n=n(\xi, \beta)$ such that $t \in \mathcal{S}_{\beta}$ for all $s \in \mathcal{S}_{\xi}$ with $n \leq s$. This shows that for every $s \in \mathcal{S}_{\xi}, q_{\mathcal{S}_{\beta}}(s)$ has cardinality at most $n$ and therefore $o\left(\mathcal{S}_{\xi} / \mathcal{S}_{\beta}\right)<n$.

(ii) Let $\xi \geq \beta$ and denote by $\delta_{\xi}$ the unique ordinal such that $\xi=\beta+\delta_{\xi}$. By (44) it suffices to show

$$
o\left(\mathcal{S}_{\xi} / \mathcal{S}_{\beta}\right) \leq \omega^{\delta_{\xi}}
$$


If $\xi=\beta$, then $\mathcal{S}_{\xi} / \mathcal{S}_{\beta}=\mathcal{S}_{\beta} / \mathcal{S}_{\beta}=\mathcal{S}_{0}$ and the result follows trivially. Let $\zeta>\beta$ and assume that $o\left(\mathcal{S}_{\xi} / \mathcal{S}_{\beta}\right) \leq \omega^{\delta_{\xi}}$ for all $\xi<\zeta$.

Suppose first that $\zeta$ is a successor ordinal and let $\xi<\omega_{1}$ be such that $\zeta=\xi+1$. Then $\delta_{\zeta}=\delta_{\xi}+1$ and $\mathcal{S}_{\zeta}=\mathcal{S}_{\xi} * \mathcal{S}_{1}$. By Lemma 40,

$$
\mathcal{S}_{\zeta} / \mathcal{S}_{\beta} \subseteq\left(\mathcal{S}_{\xi} / \mathcal{S}_{\beta}\right) * \mathcal{S}_{1}
$$

Hence by Proposition 41(i) and our inductive assumption we get

$$
o\left(\mathcal{S}_{\zeta} / \mathcal{S}_{\beta}\right) \leq o\left(\mathcal{S}_{\xi} / \mathcal{S}_{\beta}\right) \cdot o\left(\mathcal{S}_{1}\right) \leq \omega^{\delta_{\xi}} \cdot \omega=\omega^{\delta_{\zeta}} .
$$

Suppose now that $\zeta$ is a limit ordinal and let $\left(\zeta_{n}\right)_{n}$ be the increasing sequence of ordinals associated to $\zeta$ by the definition of $\mathcal{S}_{\zeta}$. For each $n \in \mathbb{N}$ we set

$$
\mathcal{F}_{n}=\left\{t \in \mathcal{S}_{\zeta_{n}}: n \leq t\right\} .
$$

Then $\mathcal{S}_{\zeta} / \mathcal{S}_{\beta}=\bigcup_{n}\left(\mathcal{F}_{n} / \mathcal{S}_{\beta}\right)$, and therefore by Proposition 41(ii),

$$
o\left(\mathcal{S}_{\zeta} / \mathcal{S}_{\beta}\right)=\sup _{n} o\left(\mathcal{F}_{n} / \mathcal{S}_{\beta}\right) \leq \sup _{n} o\left(\mathcal{S}_{\zeta_{n}} / \mathcal{S}_{\beta}\right) .
$$

Let $n_{0}$ be the least such that $\zeta_{n} \geq \beta$ for all $n \geq n_{0}$. Then by part (i),

$$
\max _{n<n_{0}} o\left(\mathcal{S}_{\zeta_{n}} / \mathcal{S}_{\beta}\right)<\omega \text {. }
$$

By our inductive assumption $o\left(\mathcal{S}_{\zeta_{n}} / \mathcal{S}_{\beta}\right) \leq \omega^{\delta_{\zeta_{n}}}$ for each $n \geq n_{0}$. Since $\sup _{n} \zeta_{n}=\zeta$, we see that $\sup _{n \geq n_{0}} \delta_{\zeta_{n}}=\delta_{\zeta}$ and therefore

$$
\sup _{n \geq n_{0}} o\left(\mathcal{S}_{\zeta_{n}} / \mathcal{S}_{\beta}\right) \leq \sup _{n \geq n_{0}} \omega^{\delta_{\zeta_{n}}}=\omega^{\delta_{\zeta}} .
$$

By (46)-(48) we obtain $o\left(\mathcal{S}_{\zeta} / \mathcal{S}_{\beta}\right) \leq \omega^{\delta_{\zeta}}$, and the proof of the inductive step and of the lemma is complete.

Proof of Proposition 5. Let $\xi=\beta+\delta$ be countable ordinals and $k \in \mathbb{N}$. By Lemma $40,\left(\mathcal{S}_{\xi} * k\right) / \mathcal{S}_{\beta} \subseteq\left(\mathcal{S}_{\xi} / \mathcal{S}_{\beta}\right) * k$ and therefore by Proposition 41 (i) and Lemma 43, we have

$$
o\left(\left(\mathcal{S}_{\xi} * k\right) / \mathcal{S}_{\beta}\right) \leq \omega^{\delta} \cdot k
$$

which in conjunction to $(44)$ gives $o\left(\left(\mathcal{S}_{\xi} * k\right) / \mathcal{S}_{\beta}\right)=\omega^{\delta} \cdot k$ and completess the proof.

\section{References}

[AA] D. Alspach and S. A. Argyros, Complexity of weakly null sequences, Dissertationes Math. 321 (1992).

[AO] D. Alspach and E. Odell, Averaging weakly null sequences, in: Lecture Notes in Math. 1332, Springer, Berlin, 1988, 126-144.

[AD] S. A. Argyros and I. Deliyanni, Examples of asymptotic $l_{1}$ Banach spaces, Trans. Amer. Math. Soc. 349 (1997), 973-995.

[AG] S. A. Argyros and I. Gasparis, Unconditional structures of weakly null sequences, ibid. 353 (2001), 2019-2058. 
[AGR] S. A. Argyros, G. Godefroy and H. P. Rosenthal, Descriptive set theory and Banach spaces, in: Handbook of the Geometry of Banach Spaces, Vol. 2, W. B. Johnson and J. Lindenstrauss (eds.), Elsevier, 2003, 1007-1069.

[AMT] S. A Argyros, S. Mercourakis and A. Tsarpalias, Convex unconditionality and summability of weakly null sequences, Israel J. Math. 29 (1998), 157-193.

[AT] S. A. Argyros and A. Tolias, Methods in the theory of hereditarily indecomposable Banach spaces, Mem. Amer. Math. Soc. 806 (2004).

[BP] C. Bessaga and A. Pełczyński, Spaces of continuous functions IV, Studia Math. 17 (1958), 165-174.

[BS] A. Brunel and L. Sucheston, On B-convex Banach spaces, Math. Systems Theory 7 (1974), 294-299.

[El] E. Ellentuck, A new proof that analytic sets are Ramsey, J. Symbolic Logic 39 (1974), 163-165.

[E] J. Elton, Thesis, Yale Univ. 1978

[K] A. Kechris, Classical Descriptive Set Theory, Grad. Texts in Math. 156, Springer, New York, 1995.

[L] D. Leung, Symmetric sequence subspaces of $C(\alpha)$, J. London Math. Soc. (2) 59 (1999), 1049-1063.

[GP] F. Galvin and K. Prikry, Borel sets and Ramsey's theorem, J. Symbolic Logic 38 (1973), 193-198.

[G] I. Gasparis, A dichotomy theorem for subsets of the power set of the natural numbers, Proc. Amer. Math. Soc. 129 (2001), 759-764.

[GOW] I. Gasparis, E. Odell and B. Wahl, Weakly null sequences in the Banach space $C(K)$, preprint, 2004.

[MS] S. Mazurkiewicz et W. Sierpiński, Contributions à la topologie des ensembles dénombrables, Fund. Math. 1 (1920), 17-27.

[NW] C. St. J. A. Nash-Williams, On well-quasi-ordering transfinite sequences, Proc. Cambridge Philos. Soc. 61 (1965), 33-39.

[O1] E. Odell, Applications of Ramsey theorems to Banach space theory, in: Notes in Banach Spaces, H. E. Lacey (ed.), Univ. Texas Press, 1980, 379-404.

[O2] - On quotients of Banach spaces having shrinking unconditional bases, Illinois J. Math. 36 (1992), 681-695.

[PR] P. Pudlák and V. Rödl, Partition theorems for systems of finite subsets of integers, Discrete Math. 39 (1982), 67-73.

[PS] A. Pełczyński and Z. Semadeni, Spaces of continuous functions III. Spaces $C(\Omega)$ for $\Omega$ without perfect subsets, Studia Math. 18 (1959), 211-222.

[R] H. P. Rosenthal, The Banach spaces $C(K)$, in: Handbook of the Geometry of Banach Spaces, Vol. 2, W. B. Johnson and J. Lindenstrauss (eds.), 2003, 15471602.

[S] J. Silver, Every analytic set is Ramsey, J. Symbolic Logic 35 (1970), 60-64.

[T] S. Todorcevic, High-dimensional Ramsey theory and Banach space geometry, in: Advanced Course on Ramsey Methods in Analysis, CRM, 2004.

Department of Mathematics

National Technical University of Athens

Athens 15780, Greece

E-mail: sargyros@math.ntua.gr

bkanel@math.ntua.gr

Received 1 April 2005;

in revised form 1 July 2005 